\title{
1 Signatures of copy number alterations in human cancer
}

2 Christopher D. Steele ${ }^{1}$, Ammal Abbasi ${ }^{2,3,4}$, S. M. Ashiqul Islam²,3,4, Azhar

3 Khandekar ${ }^{2,3,4}$, Kerstin Haase ${ }^{5}$, Shadi Hames ${ }^{1}$, Maxime Tarabichi ${ }^{5}$, Tom Lesluyes ${ }^{5}$,

4 Adrienne M. Flanagan ${ }^{1,6}$, Fredrik Mertens ${ }^{7,8}$, Peter Van Loo ${ }^{5}$, Ludmil B.

5 Alexandrov $2,3,4,{ }^{*}$, and Nischalan Pillay ${ }^{1,6,{ }^{*}}$

6

${ }^{1}$ Research Department of Pathology, Cancer Institute, University College London, London, WC1E 6BT, UK

9 'Department of Cellular and Molecular Medicine, UC San Diego, La Jolla, CA,

10 92093, USA

113 Department of Bioengineering, UC San Diego, La Jolla, CA, 92093, USA

$12{ }^{4}$ Moores Cancer Center, UC San Diego, La Jolla, CA, 92037, USA

$13{ }^{5}$ Cancer Genomics Laboratory, The Francis Crick Institute, London, NW1 1AT, UK

$14{ }^{6}$ Department of Cellular and Molecular Pathology, Royal National Orthopaedic

15 Hospital NHS Trust, Stanmore, HA7 4LP, UK

16 'Division of Clinical Genetics, Department of Laboratory Medicine, Lund University,

17 Lund, Sweden

18 'Department of Clinical Genetics and Pathology, Division of Laboratory Medicine,

19 Lund, Sweden

"Denotes equal contributions. Correspondence and request for materials should be addressed to L2alexandrov@health.ucsd.edu and N.pillay@ucl.ac.uk. 


\section{ABSTRACT}

25 The gains and losses of DNA that emerge as a consequence of mitotic errors and

26 chromosomal instability are prevalent in cancer. These copy number alterations

27 contribute to cancer initiaition, progression and therapeutic resistance. Here, we

28 present a conceptual framework for examining the patterns of copy number alterations in human cancer using whole-genome sequencing, whole-exome sequencing, and SNP6 microarray data making it widely applicable to diverse datasets. Deploying this framework to 9,873 cancers representing 33 human cancer types from the TCGA project revealed a set of 19 copy number signatures that explain the copy number patterns of $93 \%$ of TCGA samples. 15 copy number signatures were attributed to biological processes of whole-genome doubling, aneuploidy, loss of heterozygosity, homologous recombination deficiency, and chromothripsis. The aetiology of four copy number signatures are unexplained and some cancer types have unique patterns of amplicon signatures associated with extrachromosomal DNA, disease-specific survival, and gains of proto-oncogenes such as MDM2. In contrast to base-scale mutational signatures, no copy number signature associated with known cancer risk factors. The results provide a foundation for exploring patterns of copy number changes in cancer genomes and synthesise the global landscape of copy number alterations in human cancer by revealing a diversity of mutational processes giving rise to copy number changes. 


\section{MAIN}

Genome instability is a hallmark of cancer leading to changes of the genomic DNA sequence, aneuploidy, and focal copy number alterations ${ }^{1}$. Both aneuploidy and subchromosomal copy number alterations have been previously associated with increased cell proliferation, poor prognosis, and reduced infiltration of immune cells ${ }^{2-}$ ${ }^{6}$. Aneuploidy and genome-wide structural variation may originate from mitotic slippage, spindle multipolarity, and breakage-fusion-bridge (BFB) cycles ${ }^{7}$. Besides chromosome mis-segregation, other macroevolutionary mechanisms lead to changes in genomic copy number, including whole-genome doubling (WGD), where the entire chromosomal content of a cell is duplicated ${ }^{8}$ and chromothripsis where a "genomic catastrophe" leads to clustered rearrangements and oscillating copy number ${ }^{9}$. These evolutionary events may occur multiple times at different intensities during tumour development leading to a highly complex genome ${ }^{10-12}$.

The complex structural profiles of human cancers are mirrored by the intricate patterns of somatic mutations imprinted on cancer genomes at a single nucleotide level. Previously, we developed a computational framework that allows separating these intricate patterns of somatic mutations into individual mutational signatures of single base substitutions (SBS), doublet base substitutions (DBS), and small insertion or deletions (ID) ${ }^{13,14}$. Analyses of mutational signatures have provided unprecedented insights into the exogenous and endogenous processes moulding cancer genomes at a single nucleotide level with mutational signatures attributed to exposures to environmental mutagens, failure of DNA repair, infidelity/deficiency of polymerases, iatrogenic events, and many others ${ }^{15-22}$. 
70 We recently developed a "mechanism-agnostic" approach for summarising allelespecific copy number patterns in whole genome sequenced sarcomas ${ }^{23}$ which we term copy number signatures. Other cancer subtype-specific methods for interrogating copy number patterns have been created and applied to ovarian cancer and breast cancer 24,25 . While these initial approaches have led to biological and clinical insights, there is currently no approach that allows interrogating copy number signatures across multiple cancer types and across different experimental assays. To address this gap we developed a new framework for deciphering copy number signatures across cancer types and demonstrate its applicability to whole-genome sequencing, whole-exome sequencing, and SNP6 microarray data. We identified 19 distinct copy number signatures many of which are shared across multiple histologies and others that are specific to certain cancer subtypes. Extensive computational simulations, refinement and statistical association analyses were used both to assign processes to many of these signatures and to demonstrate their biological and clinical relevance. Overall, our findings shed light on the processes of chromosomal segregation errors and provide a method to distil the ensuant complex genomic configurations.

\section{A framework for pan-cancer classification of copy number alterations}

We examined the allele-specific copy number profiles of 9,873 primary cancer samples across 33 cancer types from The Cancer Genome Atlas project (TCGA; of copy number segments, proportion of the genome displaying loss of heterozygosity ( $\mathrm{LOH}$ ) and genome doubling status vary greatly amongst cancer types (Fig. 1a-b). Nevertheless, a linear relationship was observed between the 
95 number of segments and proportion of genomic $\mathrm{LOH}$, varying from cancers with

96 diploid and copy number "quiet" genomes (e.g., acute myeloid leukaemia, thymoma,

97 and thyroid carcinoma; Fig. 1a) to cancers with highly aberrant copy number profiles

98 (e.g., ovarian carcinomas and sarcomas; Supplementary Fig. 1a-b). This linear

99 relationship fails to hold only for adrenocortical carcinoma and chromophobe renal

100 cell carcinoma both of which demonstrate enrichment of LOH without enrichment of

101 copy number segmentation (Supplementary Fig. 1a-c). Additionally, considerable

102 variability of ploidy was observed both between and within cancer types (Fig. 1 b,

103 Supplementary Fig. 1d).

104 
a

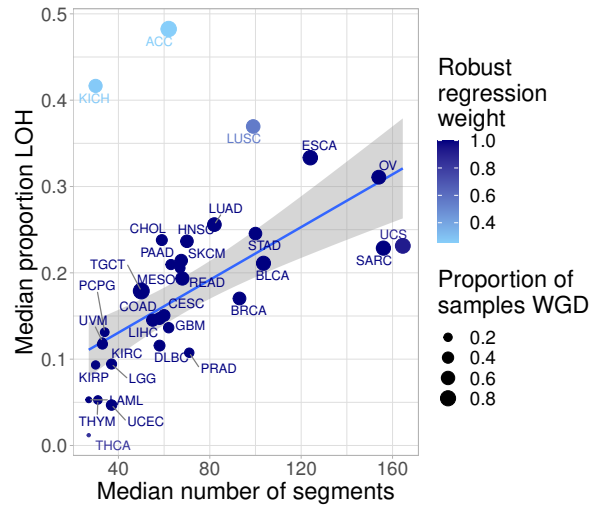

C

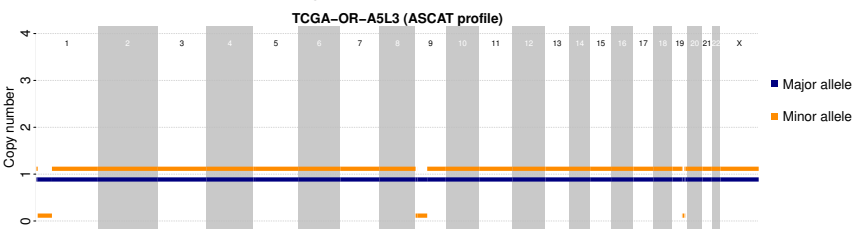

e

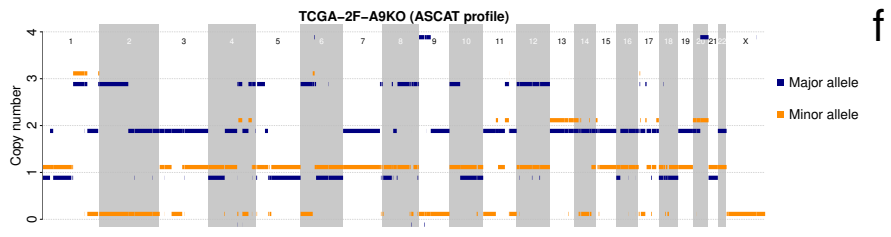

b

d
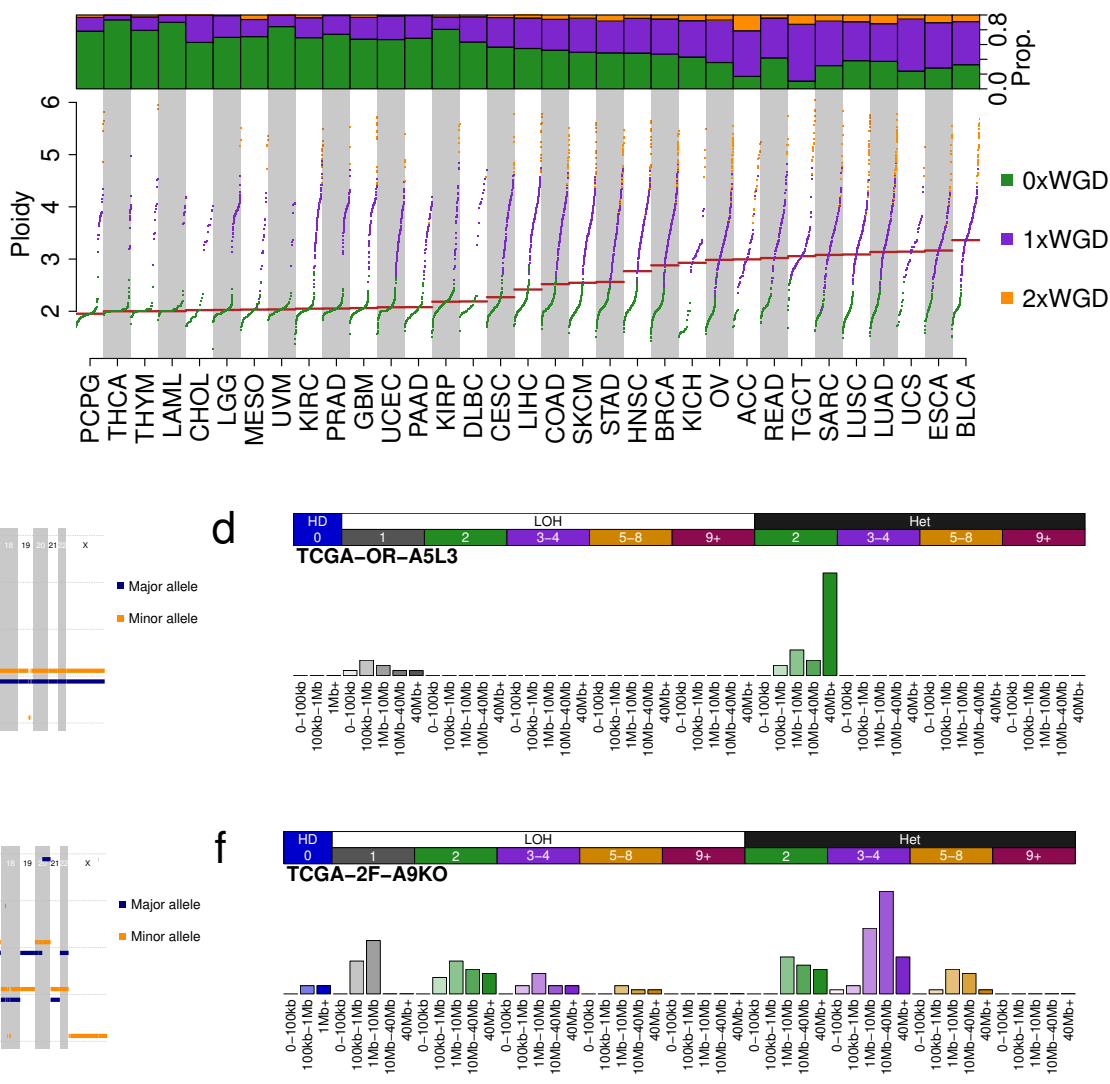

Figure 1 - Pan-cancer copy number characteristics in TCGA.

a) Copy number characteristics of 33 tumour types included in TCGA. Median number of segments in a copy number profile (x-axis), median proportion of the genome that has loss of heterozygosity (y-axis) and the proportion of samples that have undergone one or more whole genome doubling events (size). The line of best fit from a robust linear regression is shown, where the colour of points indicates the weight of the tumour type in the regression model.

b) Ploidy characteristics of all TCGA samples, split by tumour type. Bottom panel: ploidy ( $y$-axis) against quantile of ploidy (y-axis) for each sample in a tumour type, where samples are coloured by their genome doubling status: OxWGD=non genome doubled (green), 1xWGD=genome doubled (purple), 2xWGD=twice genome doubled (orange). Top panel: proportion of samples in each tumour type that are 0,1 or $2 x W G D$.

c) Allele-specific copy number profile from a majority diploid sample (sample ID: TCGA-OR-A5L3, tumour type: ACC). Copy number (y-axis) across the genome (x-axis) is given for both the major (blue) and minor (orange) allele.

d) Copy number summary for TCGA-OR-A5L3 after categorizing each of the segments. Segments are characterized first as homozygously deleted (left, blue), $\mathrm{LOH}$ (middle, white) or heterozygous (right, black), then by copy number states: $\mathrm{TCN}=0$ (blue), $\mathrm{TCN}=1$ (grey), $\mathrm{TCN}=3-4$ (purple), $\mathrm{TCN}=5-8$ (orange) and $\mathrm{TCN}=9+$ (red). Finally, segments are categorized by segment size (increasing colour saturation indicates increasing segment size): $0-100 \mathrm{~kb}, 100 \mathrm{~kb}-1 \mathrm{Mb}$, $1 \mathrm{Mb}-10 \mathrm{Mb}, 10 \mathrm{Mb}-10 \mathrm{Mb}$ and $40 \mathrm{Mb}+$ (bottom labels). Homozygous deletions have a largest segment size category of $1 \mathrm{Mb}+$.

e) Allele-specific copy number profile for a highly aberrant sample (sample ID: TCGA-2F-A9KO, tumour type: BLCA).

f) Copy number summary for TCGA-2F-A9KO. 
134 To capture biologically relevant copy number features, we developed a classification

135 framework that encodes the copy number profile of a sample by summarizing the

136 counts of segments into a 48-dimensional vector. Specifically, copy number

137 segments were classified into three heterozygosity states: heterozygous segments

138 with copy number of $\{A>0, B>0\}$ (numbers reflect the counts for major allele $A$ and

139 minor allele $B$ ), segments with $\mathrm{LOH}$ with copy number of $\{A>0, B=0\}$, and segments

140 with homozygous deletions $\{A=0, B=0\}$. Segments were further subclassified into 5

141 classes based on the sum of major and minor allele (total copy number, TCN;

142 Supplementary Fig. 1e) and chosen for biological relevance: $\mathrm{TCN}=0$ (homozygous

143 deletion), $\mathrm{TCN}=1$ (deletion leading to $\mathrm{LOH}$ ), $\mathrm{TCN}=2$ (wild type, including copy-neutral

$144 \mathrm{LOH}$ ), $\mathrm{TCN}=3$ or 4 (minor gain), $\mathrm{TCN}=5$ to 8 (moderate gain), and $\mathrm{TCN}>=9$ (high-

145 level amplification). Each of the heterozygous and LOH total copy numbers were

146 then subclassified into five classes based on the size of their segments: $0-100 \mathrm{~kb}$,

$147100 \mathrm{~kb}-1 \mathrm{Mb}, 1 \mathrm{Mb}-10 \mathrm{Mb}, 10 \mathrm{Mb}-40 \mathrm{Mb}$, and $>40 \mathrm{Mb}$ (the largest category for

148 homozygous deletions was restricted to $>1 \mathrm{Mb}$ ) in order to capture focal, large scale,

149 and chromosomal copy number changes. The segment sizes were selected to

150 ensure that a sufficient proportion of segments were classified in each category

151 resulting in a reasonable representation across the pan-cancer TCGA dataset

152 (Supplementary Fig. 1f-h). Two examples, one encoding a mostly diploid

153 adrenocortical carcinoma (Fig. 1c-d) and another encoding a genomically unstable

154 bladder cancer (Fig. 1e-f), are provided to illustrate the classification framework.

156 To determine the generalizability of our framework for pan-cancer classification of

157 copy number alterations across experimental platforms, we performed a comparative 
analysis of samples simultaneously profiled with SNP6 microarrays, whole-exome sequencing (282 samples), and whole-genome sequencing (512 samples).

160 Optimisation of the copy number calling strategy (Methods) resulted in remarkably

161 similar profiles between distinct experimental assays. Specifically, copy number

162 profiles derived from exome sequencing data had a median cosine similarity of 0.925

163 with copy number profiles derived from SNP6 microarrays (Supplementary Fig. 1i).

164 Copy number profiles derived from whole-genome sequencing data exhibited median cosine similarities of 0.933 and 0.852 with profiles derived from SNP6

166 microarrays or exome sequencing, respectively (Supplementary Fig. 1j-k). These

167 similarities are considerably better than similar comparisons observed for mutational signatures of single base substitutions derived from whole-genome and exome sequencing (median cosine similarity $=0.55)$.

The repertoire of copy number signatures in human cancer

172 Copy number profiles from SNP6 microarrays $(n=9,873)$ were concatenated into cancer type-specific matrices and separately in a global pan-cancer matrix. These matrices were decomposed using our previously established approach ${ }^{26}$ for deriving a reference set of signatures (Methods). The approach allowed the identification of both the shared patterns of copy number across all examined samples, termed, copy number signatures, as well as the quantification of the number of segments attributed to each copy number signature in each sample, termed, signature attribution.

181 By applying our copy number signature framework (Methods) we identified 19 
accurately explained the copy number profiles ( $p$-value $<0.05$, Methods) of $93 \%$ of

184 the examined TCGA samples. The remaining $7 \%$ were poorly explained due to a combination of a low number of segments and/or a high diversity of copy number

186 states in the copy number profile or few operative signatures identified

187 (Supplementary Figs. 2a-c). The 19 signatures were categorized into 6 groups based on their most prevalent features. $\mathrm{CN} 1$ and $\mathrm{CN} 2$ are primarily defined by $>40 \mathrm{Mb}$ heterozygous segments with total copy number (TCN) of 2 and 3-4 respectively. CN3 is characterized by heterozygous segments with sizes above $1 \mathrm{Mb}$ and TCN between 5 and 8 . CN4-8 each have segment sizes between $100 \mathrm{~kb}$ and

$19210 \mathrm{Mb}$ but with different TCN or LOH states. CN9-12 each have numerous LOH components with segment size $<40 \mathrm{Mb}$. CN13-14 have whole-arm or wholechromosome scale LOH events (>40Mb). CN15 consists of LOH segments with TCN between 2 and 4 as well as heterozygous segments with TCN between 3 and 8 , each with segment sizes 1-40Mb. CN16-19 exhibited complex patterns of copy

197 number alterations that are uncommon but are seen in distinct cancer types.

198 Additionally, 3 artefactual signatures (CN20-22) indicative of copy number profile over-segmentation were identified (Supplementary Fig. 2d). To determine if the copy number signatures would generalize between platforms, we compared copy number signatures derived from whole-genome and whole-exome sequencing with

202 SNP6 array signatures which showed a strong concordance with a median cosine 203 similarity between signatures above 0.80 (Supplementary Fig. 2e-h). 
a
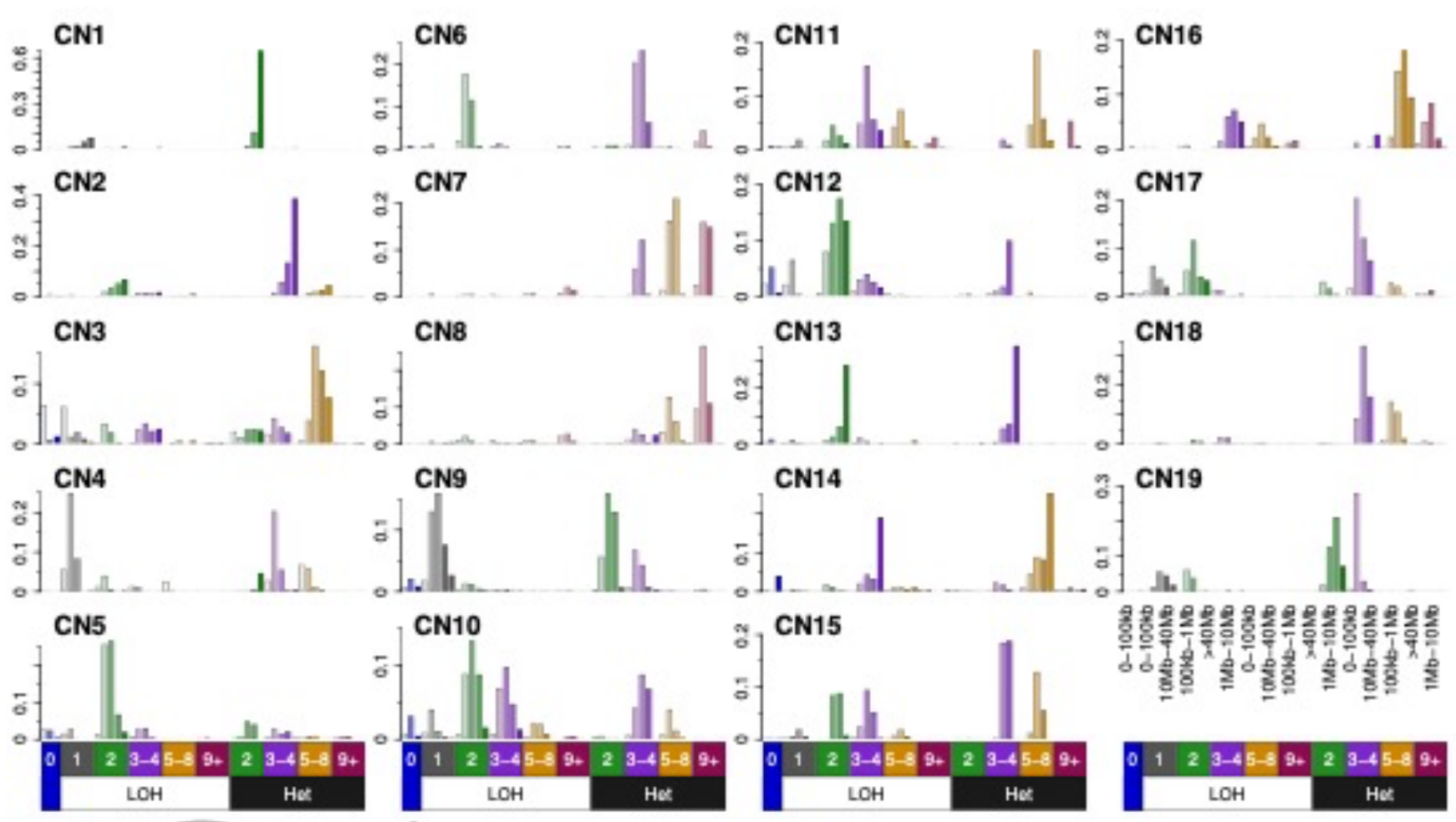

b
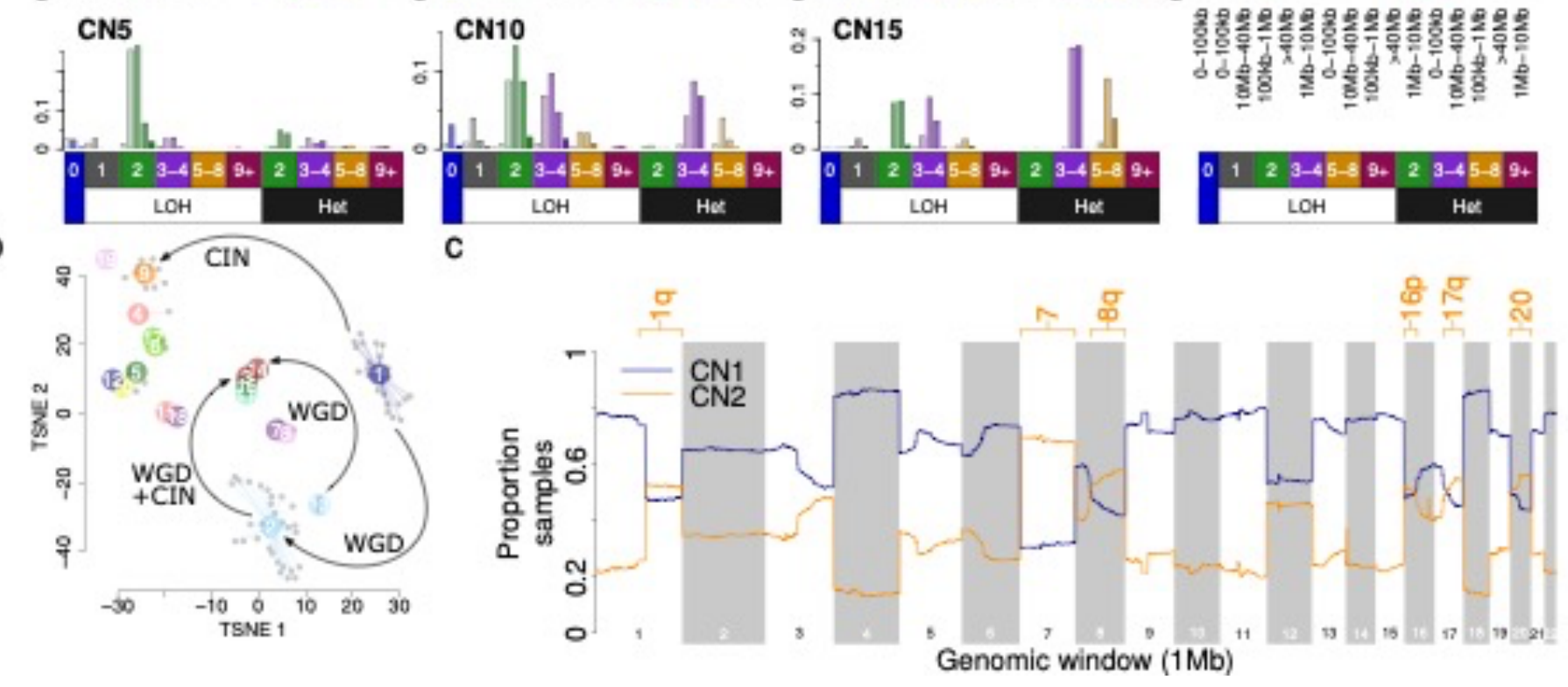

Figure 2 - Patterns of pan-cancer copy number signatures.

a) 19 identified non-artefactual copy number signatures in TCGA that are not linear combinations of any other. LOH status and total copy number are indicated below each column. Segment sizes for select bars are shown in the bottom right. Increasing saturation of colour indicates increasing segment size.

b) TSNE representation of all non-artefactual consensus signatures (colours) and the individual signatures that were combined to form each consensus signature (grey). Inferences about the relationships between signatures (see Supplementary Figure 3) are indicated with arrows; WGD=whole-genome doubling, $\mathrm{CIN}=$ chromosomal instability.

c) CN1 (blue) and CN2 (orange) recurrence (y-axis) across the genome (x-axis) in 472 highly aneuploid samples where $\mathrm{CN} 1+\mathrm{CN} 2$ attribution $=1$. Chromosome arms with $>50 \%$ samples attributed to $\mathrm{CN} 2$ are labelled. 


\section{The transitional behaviour of copy number signatures}

222 The catalogue of somatic mutations of a cancer genome is the cumulative result of

223 the mutational processes that have been operative over the lifetime of the cell from which the cancer has derived ${ }^{27}$. Analysis of SBS and ID mutational signatures have used assumptions and prior evidence that individual mutations are independent and additive $^{28}$. However, this assumption is clearly violated for large-scale macroevolutionary events such as whole-genome doubling ${ }^{29}$.

We therefore generated several synergistic lines of evidence to investigate the impact of genome doubling on copy number signatures. First, each copy number signature was tested for enrichment in non-, once- or twice-genome doubled samples (Supplementary Fig. 3a-b). Second, in silico simulations of genome doubling on the extracted signatures were performed (Methods; Supplementary

234 Fig. 3c). Third, copy number profiles arising from dynamics of whole-genome doubling and chromosomal instability (CIN) were simulated (Supplementary Fig. 3d) and re-examined for the previously derived signatures (Supplementary Fig. 3e).

238 By combining the preceding set of experiments, we revealed a transitional behaviour 239 of copy number signatures with one signature being completely replaced by another 240 upon genome doubling (Fig. 2 b). In this model, a cancer with a diploid signature

241 (CN1), may undergo genome doubling, thus altering signature CN1 into signature

$242 \mathrm{CN} 2$, or may undergo chromosomal instability transforming signature CN1 into 243 signature CN9. Through a combination of CIN and genome doubling CN2 may also 
244 be changed to CN3. Additionally, CN13 and CN14 may be linked through genome

245 doubling, on the background of early chromosomal losses.

247 While macro-evolutionary events have a transitional effect on copy number

248 signatures, we hypothesized that smaller-scale events, such as segmental aneuploidy, may reflect an additive behaviour. To investigate this, we focused on the ploidy-associated signatures CN1 and CN2, where a combination of both signatures indicates a hyper-diploid or sub-tetraploid profile. Interestingly, each signature was found at below $50 \%$ attribution in approximately a quarter of TCGA samples, suggestive of potential aneuploidy in a considerable proportion of samples. We mapped these signatures across the cancer genomes with mixtures of attributions known patterns of aneuploidy in human cancer ${ }^{30,31}$, including gains of chromosomes

\section{The landscape of copy number signatures}

260 Next, we surveyed the distribution of the 19 signatures across the different cancer

261 types (Fig. 3). Unsurprisingly, the ploidy associated signatures CN1 and CN2 were

262 found in most samples across all cancer types with different median attributions.

263 Signatures CN4, CN7, CN10, CN16, CN18, and CN19 were derived through cancer

264 type extractions and therefore unique to uveal melanoma, breast cancer, lung squamous carcinoma, ovarian carcinoma, liver cancer and paragangliomas, respectively. Signatures CN4-8 all showed segments of high total copy number and were seen in tumour types with known prevalent amplicon events ${ }^{32}$. CN9-CN12 showed differing patterns of hypodiploidy, $\mathrm{LOH}<40 \mathrm{Mb}$ and WGD reflective of 
with the known patterns of chromosomal $\mathrm{LOH}(\mathrm{cLOH})$ seen in these cancers ${ }^{33,34}$.

272 Signature CN15 was prevalent in tumour types previously described as being enriched in the tandem duplicator phenotype (TDP) ${ }^{35}$. Different cancer lineages clustered together based on the prevalence of signatures; namely TDP, wholegenome duplication, diploid chromosomal instability, simple diploidy, and human cancer ${ }^{3,36}$.

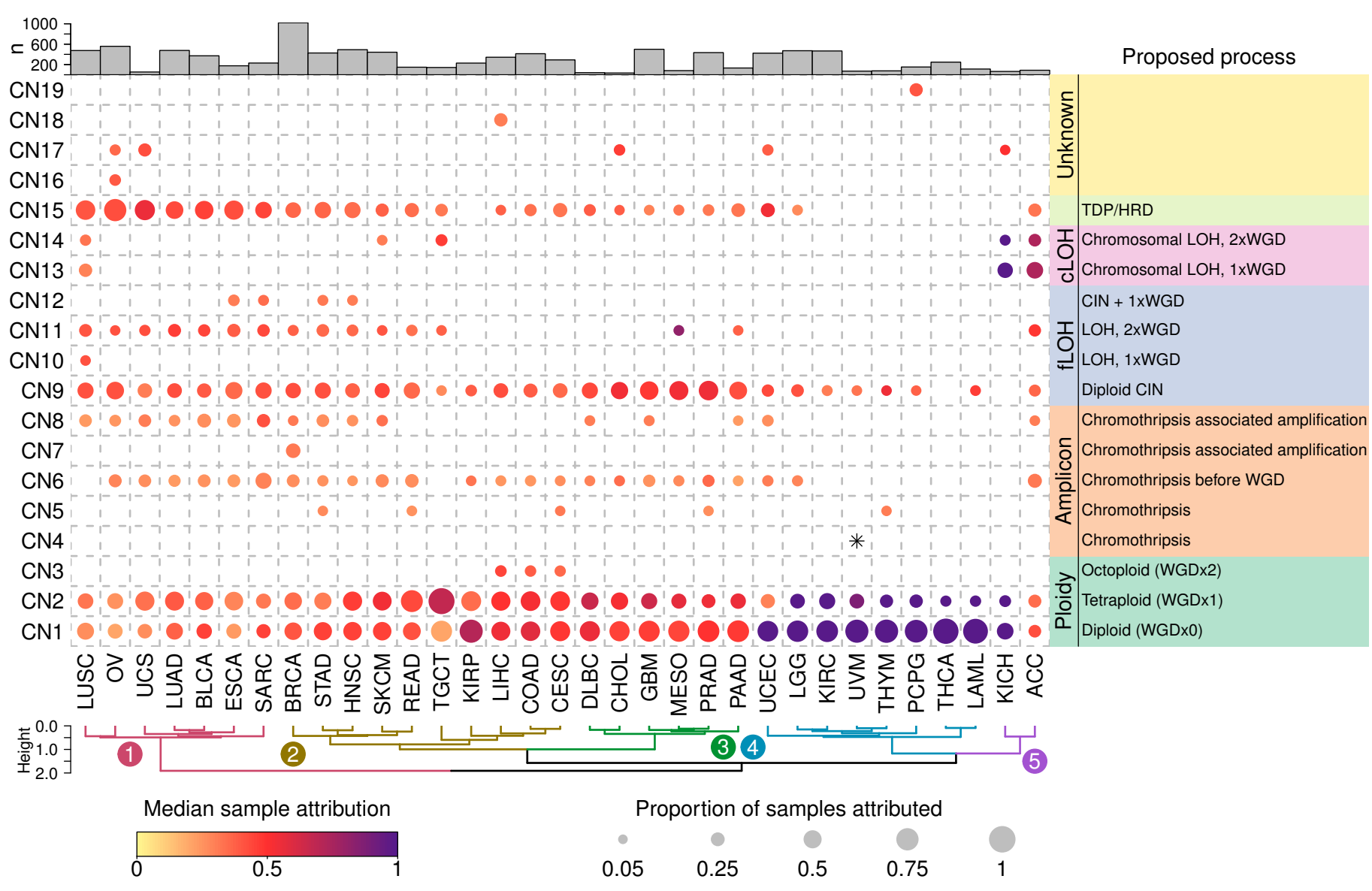


signature (size), and the median exposure of those tumours that are exposed to the signatures in each tumour type (colour). Tumour/signature combinations with less than $5 \%$ of samples exposed to the signature are not shown (except for CN4 in UVM, denoted with $a^{*}$ ). Hierarchical clustering is shown below, sample sizes are shown above. Proposed processes are shown to the right.

\section{Copy number signatures associated with amplicons}

291 Oncogene amplification has been associated with aggressive behaviour in cancer ${ }^{32}$,

292 and can originate through the processes of BFB cycles and chromothripsis ${ }^{12,37}$.

293 Reasoning that signatures with high levels of total copy number (CN4, CN5, CN6,

294 CN7, and CN8) could associate with genomic amplification we correlated these

295 signatures with known classes of amplicons ${ }^{32,38}$. All amplicon signatures were

296 positively associated with one or more amplicon types (Fig. 4a); CN8 was strongly

297 associated with all four classes of amplicon, but most strongly with extra-

298 chromosomal circular DNA amplicons (ecDNA). 


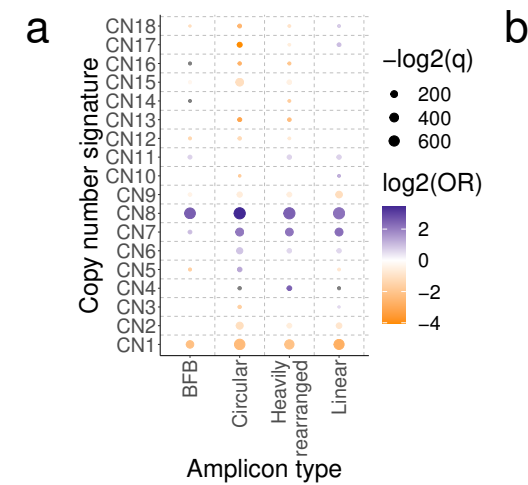

C

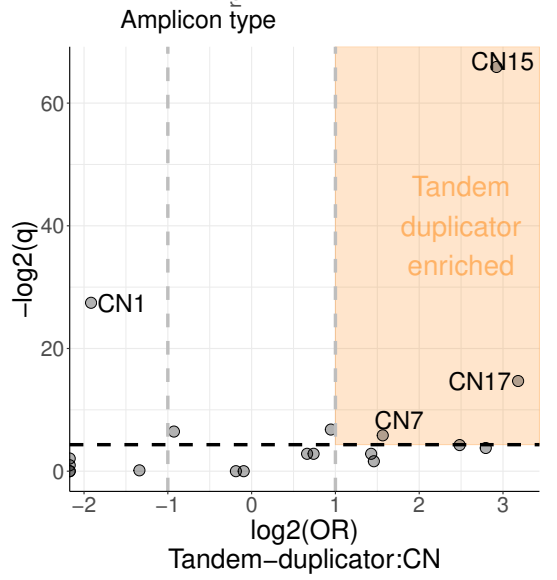

f

300

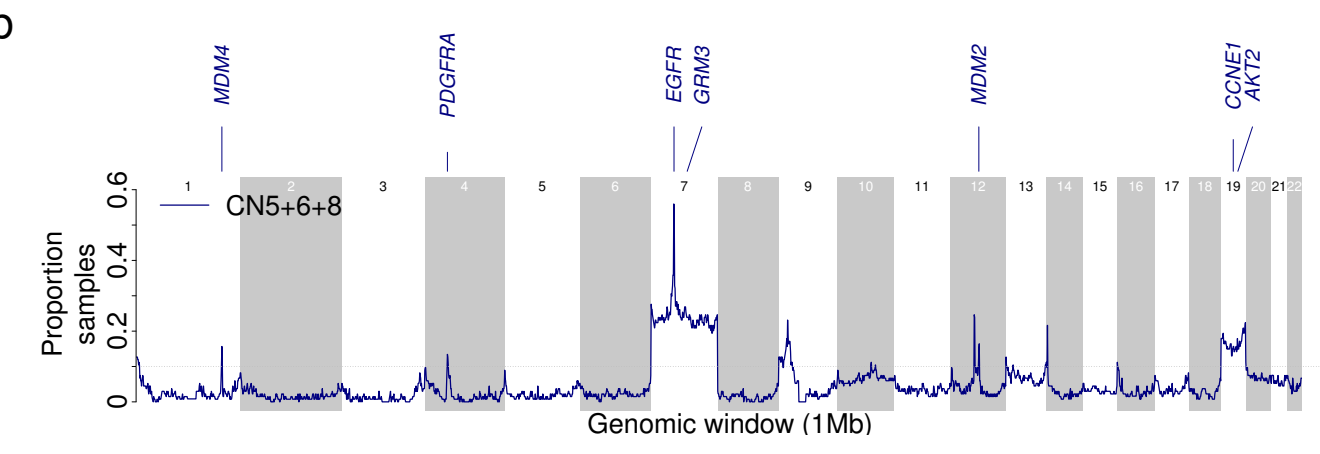

CA15 d
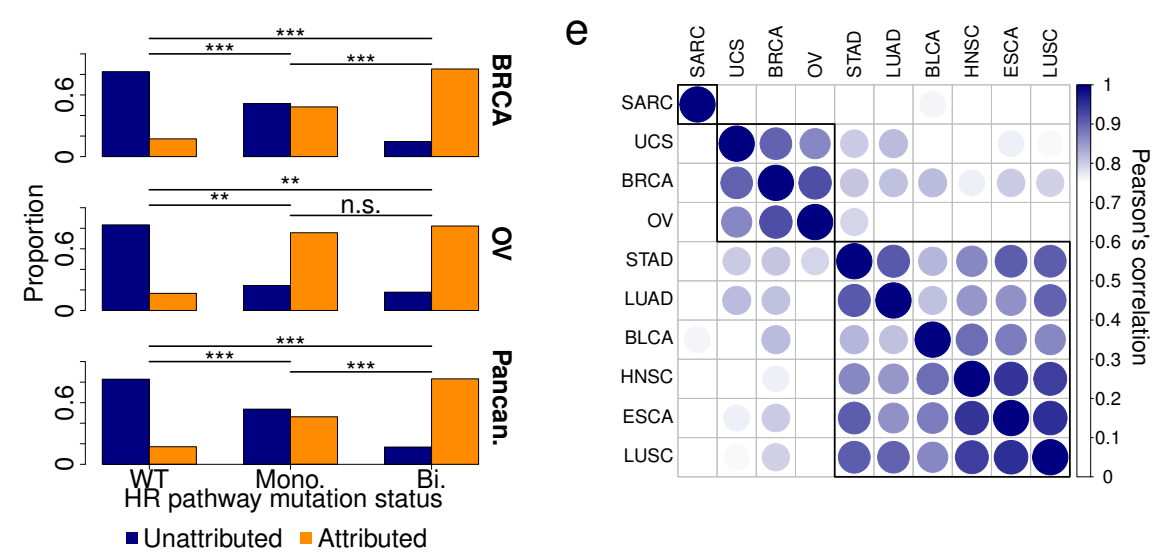

Unattributed $\backsim$ Attributed

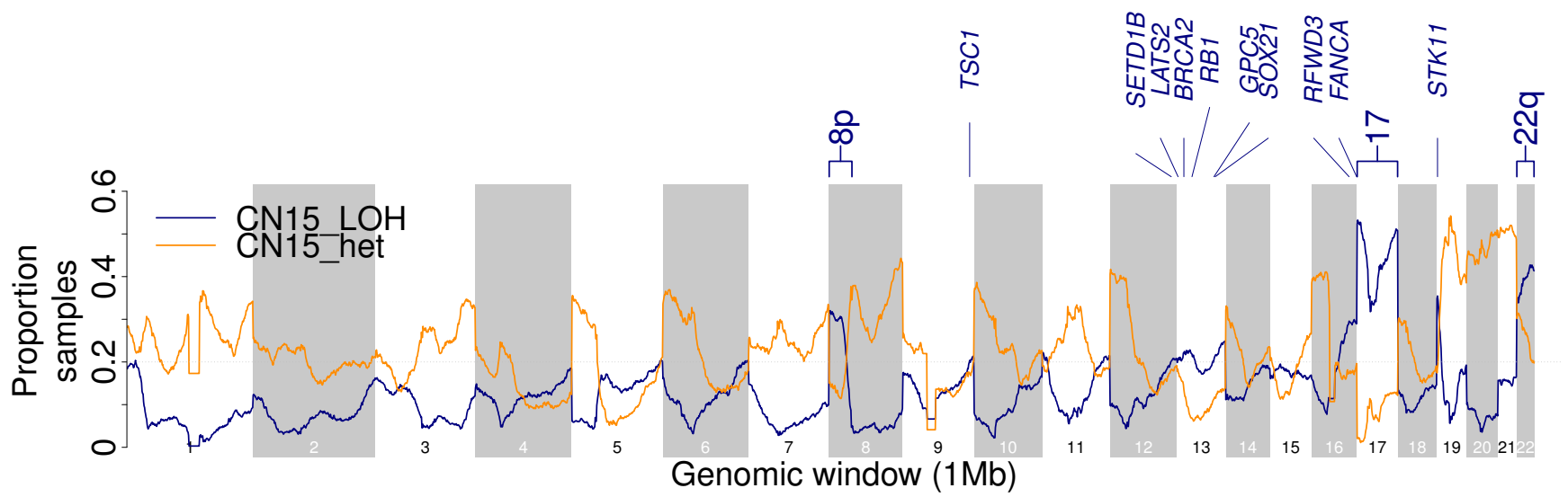

Figure 4 - Biological inference of copy-number signatures.

a) Associations between copy number signatures ( $y$-axis) and amplicon structures (x-axis), displaying the q-value (size) and log2 odds ratio (colour) from a Fisher's exact test of genomic regions attributed/not attributed to each signature against each amplicon type. Non-significant ( $q \geq 0.05)$ associations are not shown. BFB=breakage fusion bridge. CN8 was most strongly associated with circular amplicons: OR=10.8, $q<5 e-324$.

b) Recurrence of mapped amplicon signatures (CN5, CN6 and CN8) in $1 \mathrm{Mb}$ windows of the human genome across 134 GBM in which the amplicon signatures were attributed. Oncogenes in regions with $>10 \%$ samples attributed to amplicon signatures are labelled.

c) Associations between copy number signature attributed samples and tandemduplicator phenotype samples, displaying -log2(q-values) (y-axis) and log2 odds ratios (x-axis). CN15 association: $O R=7.6, q=1.5 e-20$, Fisher's exact test.

d) Correlation of CN15 attribution ( $y$-axis) with mutational status of one or more genes of the homologous recombination pathway (x-axis) in breast cancer 
(top), ovarian cancer (middle) or pan-cancer (bottom). WT=wild type. Mono = Mono-allelic and $\mathrm{Bi}=$ bi-allelic. ${ }^{*}=\mathrm{q}<0.05,{ }^{* *}=\mathrm{q}<0.01,{ }^{* * *}=\mathrm{q}<0.001$, n.s. $=\mathrm{q} \geq 0.05$.

e) Pearson's correlation of recurrence of mapping of LOH segments of CN15 to the genome calculated for all pairwise comparisons of CN15-enriched tumour types.

f) Recurrence of mapped $\mathrm{CN} 15$ in $1 \mathrm{Mb}$ windows of the human genome in all CN15 attributed BRCA, OV and UCS samples, split by LOH (blue) and heterozygous segments (orange). Tumour-suppressor genes in regions with $>20 \%$ samples attributed to CN15 with LOH segments are labelled.

Recent evidence revealed that genomic amplification can evolve through interrelated processes of chromothripsis, BFB and ecDNA formation ${ }^{11}$. Therefore, we mapped the $\mathrm{CN}$ signatures with known regions of chromothripsis ${ }^{39}$ across the genome (Methods), revealing CN5-8 as being enriched in chromothriptic regions (Supplementary Fig. 4a). Each of these signatures are dominated by small segments, while CN7-8 are both strongly associated with amplified chromothripsis ${ }^{40}$ (Supplementary Fig. 4b) and complex chromothriptic events (Supplementary Fig. 4c). Simulations of copy number profiles incorporating processes of chromothripsis, whole-genome doubling, and chromosomal duplication (Supplementary Fig. 4d) demonstrated that $\mathrm{CN} 4$ to $\mathrm{CN} 8$ can be generated through chromothripsis-like events, and that these signatures reflect distinct life histories of tumours, such as chromothripsis before or after genome doubling (Supplementary Figs. $4 c \&$ e).

Chromothripsis and gene amplification are both independently associated with poor prognosis $^{32,41}$. Attribution of any of the five amplicon signatures in their respective cancer types resulted in a poor disease-specific survival in a univariate pan-cancer analysis (Supplementary Fig. 5a). Similarly, multiple amplicon signatures were associated with a reduced disease-specific survival in multivariate pan-cancer and cancer type analyses with consistent results from analyses based on Cox-model hazard ratios (Supplementary Fig. $\mathbf{5 b}$ - $\mathbf{c}$ ) and analyses based on accelerated failure 
347 times (Supplementary Fig. 5d-e). Cancer type-specific survival analysis revealed

348 that patients with glioblastoma with operative signature CN5 had a poor disease-

349 specific survival (172 days reduced median survival; Supplementary Figure 5d). To

350 determine the topographic localization of the amplification events, we mapped the

351 amplicon signatures operative in glioblastoma (CN5, CN6, and CN8) across the

352 genome which revealed recurrence of regions involving EGFR, PDGFRA and MDM2

353 (Fig. 4b) in keeping with previous reports of chromothripsis-associated amplification

354 of these genes ${ }^{42}$.

\section{Copy number signatures associated with loss of heterozygosity}

Loss of heterozygosity $(\mathrm{LOH})$ is an important mechanism contributing to the

inactivation of tumour suppressor genes during cancer development ${ }^{39,43,44}$. We found

that 7 signatures positively correlated with $\mathrm{LOH}$ regions of the genome

360 (Supplementary Fig. 6a). Four of these signatures (CN9-12) were designated focal

$361 \mathrm{LOH}(\mathrm{fLOH})$ signatures as they exhibited predominant segments sizes $<40 \mathrm{Mb}$ (Fig.

2). The four fLOH signatures were recurrently found around tumour suppressor genes (Supplementary Fig. 6b).

In adrenocortical carcinoma and chromophobe renal cell carcinoma a characteristic pattern of chromosome-level LOH leads to hypodiploidy ${ }^{45,46}$. We identified 2 signatures (CN13 and CN14) of chromosomal-scale LOH, each of which was enriched in both of these cancers (Supplementary Fig. 6c-d). Mapping of these signatures to the genome revealed recurrent $\mathrm{LOH}$ in chromosome regions $1 p, 3 p$,

$3705 q, 9,10 q, 13 q$, and 17p (Supplementary Fig. 6e), matching known patterns of 371 aneuploidy in these tumours ${ }^{33,34}$ (Supplementary Fig. 6f-g). 
373 Copy number signature associated with tandem duplication and homologous

\section{4 recombination deficiency}

375 Somatic tandem duplications (TD) are commonly found in breast and ovarian

376 cancer $35,47,48$. Further, TD are strongly associated with failure of homologous

377 recombination repair of DNA double strand breaks e.g. due to defective BRCA1 or

$378 B R C A 2^{35,47,48}$. A detailed characterization of TD across cancer has revealed three

379 patterns with duplicated segments ${ }^{35}$ ranging around $10 \mathrm{~kb}, 200 \mathrm{~kb}$, or $2 \mathrm{Mb}$,

380 respectively. CN15 has a segment size distribution that overlaps with the largest of

381 these three patterns and was strongly associated with TD (Fig. 4c, OR=7.6, q=1.5e-

382 20, Fisher's exact test) and enriched in cancer types known to show TD

383 (Supplementary Fig. 7a) ${ }^{35}$.

384

385 Consistent with prior observations for TD, an enrichment of CN15 is observed for samples harbouring mono-allelic defects in the homologous recombination pathway compared to wild-type samples for breast cancer (Fig. 4d; OR=4.5 with $q=6.1 \mathrm{e}-14$;

Fisher's exact test), ovarian cancer $(O R=15.3$ with $q=5.9 e-3)$, and across all cancers $(\mathrm{OR}=4.2$ with $\mathrm{q}=2.2 \mathrm{e}-106)$. Further enrichments of $\mathrm{CN} 15$ were observed in samples with bi-allelic defects in the homologous recombination pathway compared to samples with mono-allelic defects for breast cancer (Fig. 4d; OR=6.2 with $q=6.2 e-5$; Fisher's exact test) and across all cancers $(O R=5.7$ with $q=4.3 e-16)$.

394 Prior analysis has shown that breakpoints resulting from TDs segregate non-

395 randomly in the genome ${ }^{35}$. Mapping of CN15 to the genomes of CN15-enriched cancers revealed a tumour type-specific distribution of LOH segments (Fig. 4e), but 
not of heterozygous segments (Supplementary Fig. 7 b). Breast and ovarian cancer as well as uterine carcinosarcoma displayed recurrent chromosomal LOH at 8p, 17

399 (including BRCA1 and TP53), and 22 (Fig. 4f). Focal LOH was also observed on 9q

400 around $T S C 1,13 q$ around $B R C A 2$ and $R B 1$, and 19p around STK11 (Fig. 4f). In

401 contrast CN15 attributed sarcomas display strong peaks of recurrent LOH around

402 known sarcoma tumour suppressor genes ${ }^{49}$ (CDKN2A, RB1, and TP53;

403 Supplementary Fig. 7c). The 6 other tumour types enriched in CN15 display

404 recurrent chromosomal LOH at 8p, 9p, 17p, 19p, and 21 (Supplementary Fig. $7 d$ ).

406 Copy number signatures associate with genomic features

407 To identify DNA damage repair mechanisms involved in the mutational processes

408 giving rise to copy number signatures, we evaluated the associations between the

409 activities of copy number signatures and single nucleotide level mutational

410 signatures from both exome and whole genome sequencing data (Fig. 5a). As

411 previously described SBS3 and ID6 are strongly associated with defective

412 homologous recombination repair ${ }^{14}$. SBS2 and SBS13 are associated with

413 APOBEC-mediated mutagenesis particularly seen near double stranded DNA

414 breaks ${ }^{50}$. As expected, CN15 was strongly associated with SBS3 and ID6 derived

415 from both WES and WGS data. Additionally, CN15 was associated with SBS2 and

416 SBS13 providing a putative mechanistic link between APOBEC activity and CN15 in

417 the context of TDPs. Negative associations were observed for diploid signature CN1

418 and APOBEC signatures SBS2 and SBS13 as well as for CN1 and tobacco-

419 associated signature SBS4. These results indicate that diploid cancer genomes have

420 lower APOBEC mutagenesis and that most cancers of tobacco smokers are not

421 diploid. 
bioRxiv preprint doi: https://doi.org/10.1101/2021.04.30.441940; this version posted April 30, 2021. The copyright holder for this preprint (which was not certified by peer review) is the author/funder, who has granted bioRxiv a license to display the preprint in perpetuity. It is made available under aCC-BY-NC-ND 4.0 International license. 
a

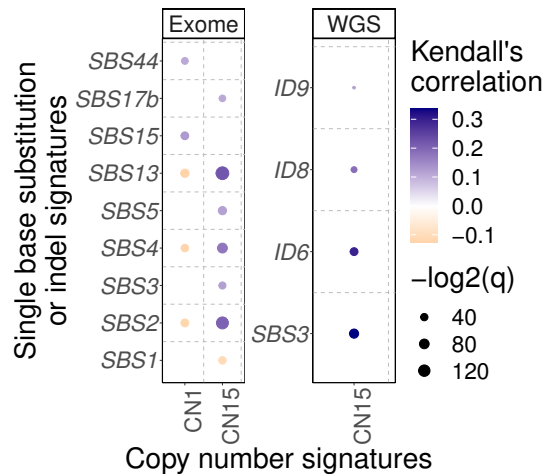

b

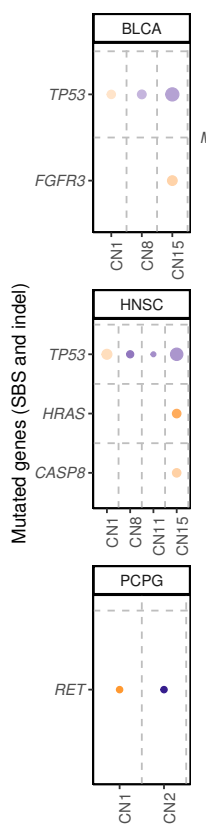

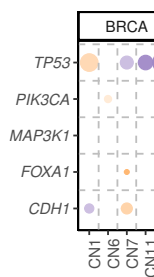
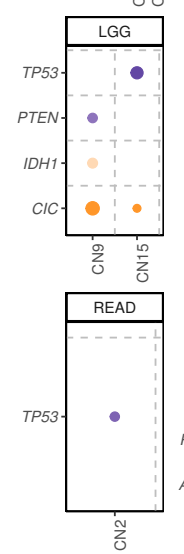

o

Copy n

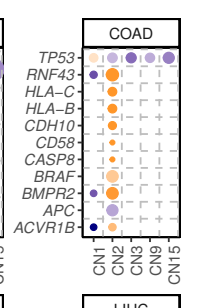

咅等言总
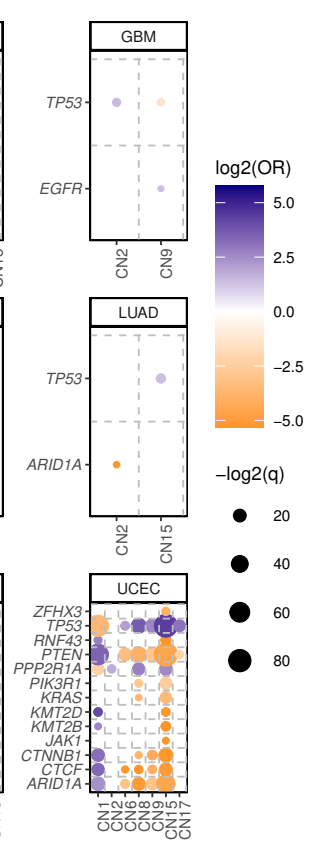

C

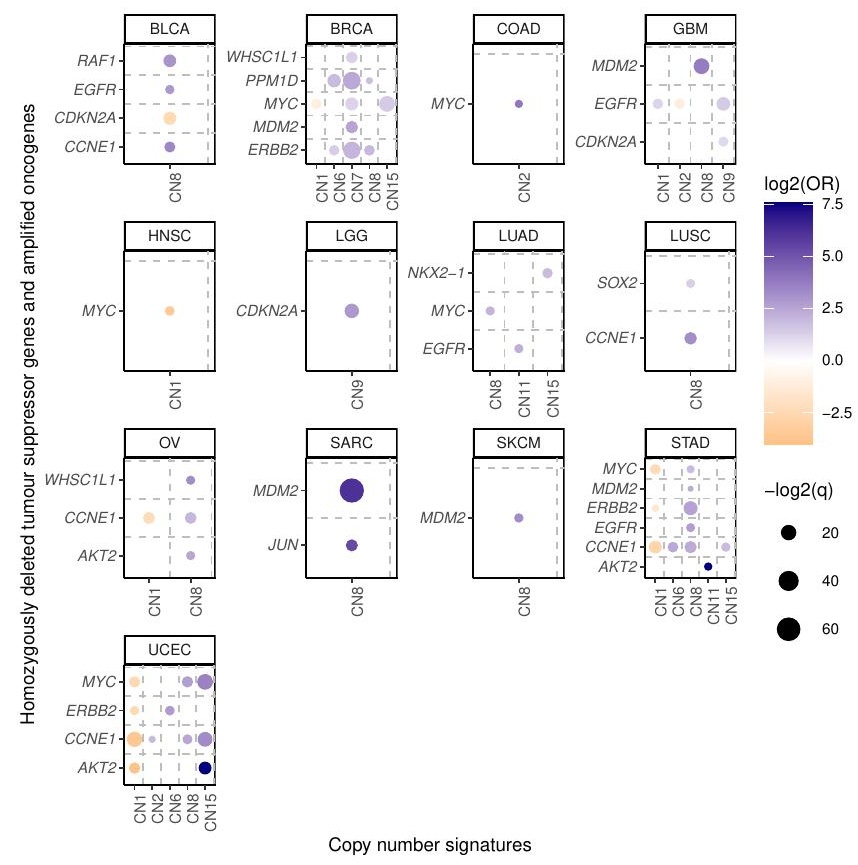

424

425

426

427

428

429

430

431

432

433

434

435

436

437

438

439

440

441

442

443

444

Figure 5 - Genomic associations of copy number signatures.

a. Correlation between copy number signature (y-axis) attribution and single base substitution signature ( $x$-axis, SBS) exposure across TCGA exomes (left) and whole genomes (right). Strength of correlation is indicated by colour (orange=anti-correlated, blue=correlated), $q$-value is indicated by size of point. Only SBS signatures with any correlation between any copy number signatures with $\mathrm{q}<0.01$ are shown. CN15 association with exome SBS3: Kendall's correlation $=0.12, \mathrm{q}=7.5 \mathrm{e}-12$. CN15 association with exome SBS2 and SBS13: Kendall's correlation $=0.2$ and $0.22, q=1.6 e-43$ and $2.2 e-50$, respectively. CN15 association with WGS SBS3: Kendall's correlation=0.34, $\mathrm{q}=1.1 \mathrm{e}-21$. CN15 association with WGS ID6: Kendall's correlation=0.29, $q=4.7 \mathrm{e}-15$.

b. Associations between copy number signatures ( $x$-axis) and driver gene $\mathrm{SNV/indel} \mathrm{status} \mathrm{(y-axis)} \mathrm{across} \mathrm{each} \mathrm{TCGA} \mathrm{tumour} \mathrm{type} \mathrm{(panels).} \mathrm{Effect} \mathrm{size}$ (log2 odds ratio, colour), and significance level (-log2 q-value, size) from a Fisher's exact test are displayed.

c. Associations between copy number signatures (x-axis) and driver gene copy number alteration status ( $y$-axis, amplification for oncogenes, homozygous deletion for tumour-suppressor genes) across each TCGA tumour type (panels). Effect size (log2 odds ratio, colour), and significance level (-log2 qvalue, size) from a Fisher's exact test are displayed. 
445 We next interrogated cancer driver gene mutations and copy number signatures and

446 found significant differences between cancer types. A consistent finding across

447 cancer was a positive association between TP53 mutation and multiple copy number

448 signatures (Fig. 5b). TP53 mutations were also associated with an increased

449 diversity of copy number signatures (Supplementary Fig. 8a; OR=3.42 with $q=1.5 e-$

450 49), supporting the link between TP53 alteration and aneuploidy ${ }^{3,51-53}$. Mutations in

451 RNF43, HLA-B, HLA-C and BRAF are commonly seen in microsatellite instable

452 (MSI) colon cancers and were found to be negatively correlated with samples with

453 tetraploid genomes (i.e., CN2 attributed; Supplementary Fig. 8b). MSI is associated

454 with high immune cell infiltration whilst aneuploidy is associated with a decrease in

455 leucocyte fraction ${ }^{54}$. Across multiple cancer types, we observe a general trend of

456 decreased leucocyte fractions in cancers with copy number signatures of aneuploidy

457 compared to diploid cancers (CN1; Supplementary Fig. 8c). Similar to colon

458 cancer, multiple cancer driver genes were associated with CN1/CN2 in endometrial

459 cancer, largely driven by differential copy number and mutation patterns seen in

460 microsatellite stable and unstable tumours (Supplementary Fig. 8d).

461

462 To assess the relationships between copy number signatures and copy number

463 driver genes, we evaluated the associations between attributions of copy number

464 signatures and homozygous deletions of COSMIC tumour suppressor genes as well

465 as between attributions of copy number signatures and amplifications of known

466 proto-oncogenes ${ }^{55}$. Copy number drivers such as MDM2, EGFR, CCNE1, MYC, and

467 ERBB2 were strongly positively associated with amplicon signatures CN6-8 as well

468 as CN15 (Fig. 5c). In contrast, CDKN2A was the only homozygously deleted tumour

469 suppressor gene associated with any signature, most commonly CN9. 
471 In contrast to single-nucleotide level SBS and ID signatures ${ }^{14}$, no associations were

472 found between any copy number signature and cancer risk factors: gender, smoking

473 status, or alcohol consumption (Supplementary Fig. 8e). Significant associations

474 were found between age and copy number signature attribution in individual tumour

475 types (Supplementary Fig 8f), however, these were driven by tumour sub-type

476 differences: serous versus endometrioid endometrial cancers (difference in mean

477 age at diagnosis $=4.7$ years, $p=9.0 e-5$, Mann-Whitney test) in which non-

478 endometrioid endometrial cancers are strongly associated with $\mathrm{HRD}^{56}$ and enriched

479 in CN15 (OR=16.7, p<7.1e-26, Fisher's exact test); synovial sarcoma versus other

480 sarcoma (difference in mean age at diagnosis=-22.3 years, $p=4.3 e-3$, Mann-Whitney

481 test) in which synovial sarcomas are karyotypically simple ${ }^{49}$ and enriched in CN1

482 (OR=Inf, $p=2.3 e-5$, Fisher's exact test). 


\section{DISCUSSION}

485 In this report, we provide the first pan-cancer framework for analysing copy number signatures as well as the first comprehensive analysis of copy number signatures in

487 human cancer. The results revealed multiple distinct copy number signatures

488 including ones attributed to ploidy, amplification, loss of heterozygosity, chromothripsis, and tandem duplications. Multiple signatures of unknown processes , cancer subtype specific signatures as well as artefactual signatures were identified.

491 Unlike SBS and ID mutational signatures, copy number signatures did not associate with known cancer risk factors. Rather, copy number signatures reflect the activity of endogenous mutational processes such as homologous recombination deficiency, aberrant mitotic DNA replication, and chromothripsis ${ }^{11,12}$.

The field of copy number signatures is nascent, with three distinct methods previously implemented in three distinct tumour types ${ }^{23-25}$. As the field matures it will become increasingly clear which models are better suited to addressing specific clinical or biological questions. To resolve these questions, pan-cancer analyses utilizing all of these methods will be key, and we present here the first step towards that goal; a mechanism-agnostic pan-cancer compendium of allele-specific copy number signatures.

\section{ACKNOWLEDGEMENTS}

NP is a Cancer Research UK Clinician Scientist fellow (Award - 18387). CDS 
and the Cancer Research UK University College London Experimental Cancer

Medicine Centre.

511 Alexandrov laboratory was supported by US National Institute of Health's R01

512 ES030993 and R01 ES032547. LBA is an Abeloff V Scholar and he is supported by

513 an Alfred P. Sloan Research Fellowship. Research at UC San Diego was also supported by a Packard Fellowship for Science and Engineering to LBA.

516 This work was supported by the Francis Crick Institute, which receives its core

517 funding from Cancer Research UK (FC001202), the UK Medical Research Council

518 (FC001202), and the Wellcome Trust (FC001202). For the purpose of Open Access,

519 the authors have applied a CC BY public copyright licence to any Author Accepted

520 Manuscript version arising from this submission. This project was enabled through

521 access to the MRC eMedLab Medical Bioinformatics infrastructure, supported by the

522 Medical Research Council (grant number MR/L016311/1). PVL is a Winton Group

523 Leader in recognition of the Winton Charitable Foundation's support towards the establishment of The Francis Crick Institute.

525 Compute resources were provided by UC San Diego through the Triton Shared

526 Computing Cluster, and by UCL through the Myriad computing cluster.

528 The results shown here are in whole or part based upon data generated by the

529 TCGA Research Network: https://www.cancer.gov/tcga.

531 Thanks to Dr Marnix Jansen and Dr Hamzeh Kayhanian for critical input to the work 532 shown here. 


\section{CONFLICTS OF INTEREST}

536 LBA is an inventor of a US Patent $10,776,718$ for source identification by non-

537 negative matrix factorization.

\section{AUTHORS CONTRIBUTIONS}

540 Study was conceived and designed by CDS, NP and LBA. Data analysis was

541 performed by CDS, AA, SMAI, AK, KH, SH, MT and TL. Manuscript was written by

542 CDA, NP and LBA. Interpretation of data and contributions to writeup were provided

543 by MT, TL, AMF, FM and PVL.

\section{DATA AVAILABILITY}

546 No new data was generated for this study. ASCAT copy number profiles that were

547 generated for a different study and analysed here can be found at:

548 https://github.com/VanLoo-lab/ascat/tree/master/ReleasedData/TCGA SNP6 hg19

\section{CODE AVAILABILITY}

551 Code for summarising copy number profiles into 48-length vectors can be found at:

552 https://github.com/AlexandrovLab/SigProfilerMatrixGenerator

553 Code for extracting copy number signature can be found at:

554 https://github.com/AlexandrovLab/SigProfilerExtractor

555 Code for decomposing copy number summaries into known copy number signatures

556 can be found at:

557 https://github.com/AlexandrovLab/SigProfilerSingleSample

558 Bespoke scripts for all other analysis are available from authors upon request. 


\section{ONLINE METHODS}

\section{$561 \quad$ Utilized datasets}

562 Using SNP6 microarray data, copy number profiles were generated for 9,873

563 cancers and matching germline DNA of 33 different types from The Cancer Genome

564 Atlas (TCGA) $)^{43}$ using allele-specific copy number analysis of tumours (ASCAT) ${ }^{58}$

565 with a segmentation penalty of 70 (Supplementary Table 1). Additionally, a set of

566 whole-genome sequences from 512 cancers of the International Cancer Genome

567 Consortium (ICGC) that overlapped with tumour profiles in TCGA were analysed ${ }^{39}$ to

568 generate WGS-derived copy number profiles(see below). Lastly, a set of wholeexome sequences from 282 cancers from TCGA was analysed to generate exomederived copy number profiles (see below).

\section{Copy number profile summarization}

573 Copy number segments were categorized into three heterozygosity states: heterozygous ( $C N=\{>0,>0\}$ for the major and minor alleles respectively), loss of heterozygosity $(\mathrm{LOH} ; \mathrm{CN}=\{>0,0\})$ and homozygous deletion $(\mathrm{CN}=\{0,0\})$. Segments were further subclassified into 5 categories of total copy number: CNO reflects

577 homozygous deletions, CN1 represents a genomic deletion, CN2 represents a diploid state, CN3-4 is a tri-to-tetraploid or gained state, CN5-8 is a penta-tooctoploid state and CN9+ represents high-level amplifications. Segments were

580 further subclassified into 5 size categories: $0-100 \mathrm{~kb}, 100 \mathrm{~kb}-1 \mathrm{Mb}, 1 \mathrm{Mb}-10 \mathrm{Mb}, 10 \mathrm{Mb}-$

$58140 \mathrm{Mb}$, and $>40 \mathrm{Mb}$. For homozygous deletions only 3 size categories were used: 0-

$582100 \mathrm{~kb}, 100 \mathrm{~kb}-1 \mathrm{Mb}$, and $>1 \mathrm{Mb}$. In this way copy number profiles were summarized as counts of 48 combined copy number categories defined by heterozygosity, copy number and size, which we will define as $N=\left[n_{1}, n_{2}, \ldots, n_{48}\right]$. For a given dataset, 
the copy number profiles of a set with $S$ samples are then summarized as a nonnegative matrix with $S \times 48$ dimensions.

\section{Deciphering signatures of copy number alterations}

Copy number signatures were extracted by applying our previously developed

approach for creating a reference set of signatures ${ }^{14}$. Specifically,

591 SigProfilerExtractor $v 1 \cdot 0.17^{26}$ was applied to the matrix encompassing all TCGA

592 samples as well as separately to each matrix corresponding to an individual tumour

593 type. In brief, SigProfilerExtractor utilizes nonnegative matrix factorization (NMF) to

594 find a set of copy number signatures ranging from 1 to 25 components for each

initializations of the lower dimension matrices were performed on the Poisson

resampled data. SigProfilerExtractor was used with default parameters, except for

598 the initializations of the lower dimension matrices where random initialization was

utilized consistent with our prior analyses of mutational signatures ${ }^{14,59}$ After

600 performing 250 nonnegative matrix factorizations, SigProfilerExtractor clusters the factorization within each decomposition to automatically identify the optimum number of operative signatures that best explain the data without overfitting these data ${ }^{26}$.

As previously done ${ }^{60}$, the sets of all identified copy number signatures were combined into a reference set of pan-cancer copy number signatures by leveraging hierarchical clustering based on the cosine dissimilarities between each signature.

607 The number of combined signatures is chosen to maximise the minimum average cosine similarity between each signature in a cluster and the mean of all samples in 
610 similarity to the combined copy number signature for that cluster. Simultaneously,

611 the maximum cosine similarity between mean copy number signatures for each

612 cluster is minimized, to ensure that each combined signature is distinct from all

613 others. To avoid reference signatures being linear combinations of two or more other

614 signatures, for each identified signature, a synthetic sample was created with the

615 pattern of the signature multiplied by 1,000 copy number segments. Further, the

616 synthetic sample was resampled with probabilities $p_{i, f}=d_{i, f} / \sum_{j=1}^{48} d_{j, f}$, where $d_{i, f}$ is

617 the strength of the $i^{\text {th }}$ copy number category in the $f^{\text {th }}$ identified signature. Each

618 resampling was then scanned for activity of all other signatures from the reference

619 set. If a resampled sample can be reconstituted with a cosine similarity $>0.95$ by 3 or

620 fewer other signatures, the signature used to create the synthetic sample was

621 deemed to be a linear combination of those signatures, and the signature was

622 removed from the global reference set of signatures.

\section{Reference set of copy number signatures}

625 Initially 28 pan-cancer copy number signatures were derived from the different

626 SigProfilerExtractor analyses of the 9,873 copy number profiles from SNP

627 microarrays. In silico evaluation and manual curation showed that 10 copy number

628 signatures were linear combinations of two or more other signatures. Additionally, 3

629 signatures were deemed to be artefactual due to over-segmentation of copy number

630 profiles. These artefactual signatures were removed from further analyses, as were

631 the samples with any attribution of any of these artefactual signatures (116 samples;

$6321.2 \%$ of all TCGA samples). Moreover, samples with $>25 \mathrm{Mb}$ of homozygous

633 deletions across the genome were removed from downstream analysis (58

634 samples), leaving 9,699 samples for full analysis. Upon signature assignment (see 
below) 3 of the signatures that were removed due to linear combination were reextracted within tumour-type specific assignment (cosine similarity=1), suggesting some copy number profiles could not be explained well without these 3 signatures.

As a result, these 3 signatures were reintroduced into the compendium of signatures, leaving a total of 19 non-artefactual pan-cancer signatures of copy number alteration.

CN1-3 form a group of ploidy-associated signatures. CN1 and CN2 display TCN between 2 and 3-4 respectively, with predominantly $>40 \mathrm{Mb}$ heterozygous segments. CN3 consists of predominantly heterozygous segments of TCN 5-8 with sizes $>1 \mathrm{Mb}$.

CN4-8 form a group of amplicon-associated signatures, that all have segment sizes predominantly between $100 \mathrm{~kb}$ and $10 \mathrm{Mb}$ but with differing TCN or LOH states. CN4 consists of a mixture of LOH segments with TCN 1 and heterozygous segments with TCN 3-4. CN5 consists almost entirely of LOH segments with TCN 2. CN6 consists of a mixture of LOH segments with TCN 2 and heterozygous segments with TCN 34. CN7 consists of a mixture of heterozygous segments with TCN of 3-4, 5-8 and 9+. CN8 consists of predominantly heterozygous segments with TCN 9+.

654 CN9-12 form a group of signatures with considerable LOH components. CN9 consists of a mixture of LOH segments with TCN 2 and heterozygous segments with

$656 \mathrm{TCN} 2$, each ranging from $100 \mathrm{~kb}-40 \mathrm{Mb}$. CN10 consists of a mixture of LOH segments with TCN 2 and 3-4 as well as heterozygous segments with TCN 3-4 between $100 \mathrm{~kb}$ and $40 \mathrm{Mb}$. CN11 consists of a mixture of LOH segments with TCN 
consists of mostly LOH segments of TCN 2 with sizes above $100 \mathrm{~kb}$ and additional heterozygous segments of TCN 3-4 with sizes between 10 and 40Mb.

662

663 CN13-14 form a group of signatures with whole-arm or whole-chromosome scale

664 LOH events. CN13 consists of LOH segments with TCN 2 and heterozygous segments with TCN 3-4, each at $>40 \mathrm{Mb}$, while CN14 is similar but with TCN 3-4 and 5-8 for LOH and heterozygous segments respectively.

CN15 has been associated with the tandem duplicator phenotype (Fig. 4). This signature consists of LOH segments of TCN 2 and 3-4 as well as heterozygous segments of TCN 3-4 and 5-8, each with segment sizes 1-40Mb.

CN16-19 originate from unknwon processes and are diverse in their copy number patterns. CN16 consists of predominantly heterozygous segments of TCN 4-8 at $>1 \mathrm{Mb}$, but with appreciable contributions of LOH segments with TCN 3-4 at $>1 \mathrm{Mb}$ and heterozygous segments with TCN 9+ at >100kb. CN17 consists of segments between $100 \mathrm{~kb}$ and $40 \mathrm{Mb}$ that are heterozygous with TCN 3-4 or less commonly LOH with TCN 1 or 2 . CN18 consists of predominantly heterozygous segments with TCN 3-4 at $100 \mathrm{~kb}-40 \mathrm{Mb}$ with some heterozygous segments of TCN 3-4 at $100 \mathrm{~kb}-$ 10Mb. CN19 consists of heterozygous segments with TCN 2 at $>1 \mathrm{Mb}$ and many 680 heterozygous segments with TCN 3-4 at 100kb-1Mb.

\section{Assignment of copy number signatures to individual cancer samples}

683 The global reference set of copy number signatures was used to assign an activity 684 for each signature to each of 9,873 examined samples using the decomposition 
module of the SigProfilerExtractor ${ }^{26}$. For the assignment, the information of the de novo signature and their activities assigned to each sample were used to implement

687 the decomposition module with default parameters except for the NNLS addition penalty (nn/s_add_penalty) which was set to 0.1 , the NNLS removal penalty

689 (nnls_remove_penalty) which was set to 0.01 , and the initial removal penalty

690 (initial_remove_penalty) which was set to 0.05. Signatures were assigned to

691 samples in both tumour-specific evaluations and in a pan-cancer evaluation. As

692 previously done ${ }^{60}$, the signature attributions from either tumour-specific or pan-

693 cancer evaluations that gave the best cosine similarity between the input sample

694 vector and the reconstructed sample vector were used as the attributions for that

695 sample in all subsequent analyses.

697 Copy number signatured derived from whole-genome and exome sequencing

\section{8 data}

699 A set of samples from TCGA with both SNP-array and exome sequencing data were 700 selected $(n=282)$. Copy number profiles were generated from the exome sequencing data using ASCAT across all of the dbSNP common SNP positions with a segmentation penalty ranging from 20 to 140 . Signatures were re-extracted for these 282 samples from both the SNP-array derived copy number profiles and the exomederived copy number profiles, and the resulting signatures were compared.

For whole-genome sequencing data, we examined 512 whole-genome sequenced samples from the PCAWG project overlapping with TCGA samples with microarray data. Copy number profiles from whole-genome sequencing data were generated using ASCAT across the SNP6 positions, with a segmentation penalty ranging from 
71020 to 120. Signatures were extracted for samples with both SNP6 microarray derived

711 copy number profiles and the WGS derived copy number profiles, and the extracted

712 signatures were compared. In all cases, segmentation penalty of 70 gave the best

713 concordance for both copy number profiles and extracted copy number signatures

714 based on SNP6 microarray, whole-genome sequencing, and whole-exome

715 sequencing data.

716

717 Mapping copy number signatures to the landscapes of cancer genomes

718 Given the original copy number profiles, the identified signature matrix of $c$ copy

719 number classes by $f$ signatures, and the signature activity matrix of $s$ samples by $f$

720 signatures, it is then possible to map signatures to the genomic landscape for each

721 cancer sample. The probability of each copy number class, $\boldsymbol{c}$, having originated from

722 each signature, $\boldsymbol{i}$ from a total of $\boldsymbol{I}$ signatures, in a sample $\boldsymbol{j}$ can be defined as:

$$
m_{i, j, c}=\frac{\boldsymbol{f}_{c, i} \boldsymbol{e}_{i, j} \boldsymbol{l}_{j}}{\sum_{k=1}^{I} \boldsymbol{f}_{c, k} \boldsymbol{e}_{k, j} \boldsymbol{l}_{j}}
$$

724

where $\boldsymbol{f}$ is the normalised signature matrix, $\boldsymbol{e}$ is the normalized attribution matrix,

and $\boldsymbol{l}$ is a matrix of the number of segments in the copy number profile of each

sample. The likelihood of each signature contributing to a given genomic window,

727 here taken as each chromosome, is then the sum of copy number class probabilities

728 for each segment in that window:

$$
p_{i, j, w}=\sum_{x=1}^{l_{j, w}} m_{i, j, c_{x}}
$$

730 Once these chromosome likelihoods have been calculated, the individual segments

731 in a chromosome are assigned to their maximum likelihood signature. Once copy

732 number signatures have been mapped to the genome at a segment level, it is

733 possible to interrogate the recurrence of signatures across the genome for a given 

each signature in each window.

Associations between copy number signatures and events defined by genomic region

741 Localised events (chromothripsis ${ }^{39}$ and amplicon structure ${ }^{38}$ ) identified using WGS data were associated with mapped copy number signatures from TCGA for all available matching samples (chromothripsis $n=657$; amplicon $n=1703$ ). Each segment in every sample was categorised as overlapping or non-overlapping of a localized event. For each copy number signature, the association was then tested using a two-sided Fisher's exact test on a contingency table of segments categorized as overlapping or non-overlapping of a localized event and assigned to or not assigned to the given copy number signature, across all samples. Multiple-testing correction was performed using the Benjamini-Hochberg method.

\section{Genome doubled copy number signatures}

With the copy number categories being defined as $0,1,2,3-4,5-8$, and $9+$, it is possible to artificially 'genome double' any copy number category, other than 0 , by assigning it to the next highest copy number category. In this way we artificially 'genome doubled' each signature by assigning the count for each copy number class to its next highest copy number class. First, the copy number 1 class is assigned a count of 0 , then each copy number class is assigned the count of the preceding copy 
number class of 1, 3-4 assigned previous 2 , etc., until finally the copy number $9+$ class is assigned a count that is the sum of the previous copy number $5-8$ class and 9+ class. During this conversion, $\mathrm{LOH}$ and size categories are retained, so that the only shift is in copy number. Having performed this conversion, cosine similarities between the artificially 'genome doubled' signatures and the original signatures were calculated. Any genome-doubled and original signature pair that had a cosine similarity $>0.85$ was considered to contain a pair of signatures with analogous copy number patterns distinguished only by their genome doubling status.

\section{Associations between copy number signatures and ploidy}

Ploidy for each copy number profile was calculated as the relative length weighted displayed LOH (pLOH) were also calculated. Samples with a ploidy above $3 / 2^{*} \mathrm{pLOH}+3$, meaning an $\mathrm{LOH}$-adjusted ploidy of 3 or greater were deemed to be genome doubled samples, while samples with a ploidy above $-5 / 2^{*} \mathrm{pLOH}+5$, meaning an LOH-adjusted ploidy of 5 or greater, were deemed to be twice genome doubled samples. All other samples were considered as non-genome doubled samples. Each signature (CN1-19) was associated with each genome doubling category (GDx0, GDx1, and GDx2) using a one-sided Fisher's exact test on a contingency table with samples categorized by whether the samples have $>0.05$ attribution to the given copy number signature or not, and whether the sample has

780 the given genome doubled category or not. All p-values were corrected for multiple hypothesis testing using the Benjamini-Hochberg method. 


\section{Associations between copy number signatures and known cancer risk factors}

784

785

786

787

788

789

790

791

792

793

794

795

796

797

798

799

800

801

802

803

804

805

806

807

Associations between attributions of copy number signatures and attributions of single-base substitutions, indels, and doublet base signature exposures ${ }^{14}$ were performed using Kendall's rank correlation. Only the significant associations found in both cancer-type specific and pan-cancer analysis were reported. For the cancer risk association analyses, copy number signatures were associated with gender ${ }^{61}$, tobacco smoking ${ }^{18}$, and alcohol drinking status ${ }^{62}$. For each copy number signature, the association was conducted using a two-sided Fisher's exact test on a contingency table of a clinical feature categorized as present or absent and assigned to or not assigned to the given copy number signature across all samples. All pvalues were corrected for multiple hypothesis testing using the Benjamini-Hochberg method.

Associations between copy number signature attribution (binarized to present or absent) and the tandem duplicator phenotype (also binarized to present or absent) ${ }^{35}$ were performed using a two-sided Fisher's exact test $(n=882)$. This was performed for each copy number signature separately. All p-values were corrected for multiple hypothesis testing using the Benjamini-Hochberg method and only associations with $q<0.05$ were reported.

Associations between copy number signature attribution (binarized to present or absent) and driver gene SNV/indel mutation status ${ }^{63}$ were performed within tumour types using a two-sided Fisher's exact test ( $n=6,543$ across all cancer types). This was performed for all copy number signature/gene combinations for which the gene was mutated in the given cancer type and the copy number signature was observed 
in the given cancer type. All p-values were corrected for multiple hypothesis testing $|\log 2(\mathrm{OR})|>1$ were reported.

812 Driver copy number alterations of COSMIC cancer gene census genes ${ }^{55}$ were

813 defined as: (i) homozygous deletion $(\mathrm{CN}=\{0,0\})$ of genes listed as deleted (D) in

814 COSMIC mutation types; or (ii) amplification $\left(\mathrm{CN}>2^{*}\right.$ ploidy+1) of genes listed as amplified (A) in COSMIC mutation types. Associations were then performed on copy

816 number driver alterations for SNV/indel driver gene alterations as above $(n=9,699$

817 across all cancer types).

819 The diversity of copy number signatures, as defined by Shannon's diversity index, was associated with both SNV/indel and copy number driver gene mutations using a

821 logistic regression model with binary diversity $\{>0,=0\}$ as the dependent variable, 822 and tumour type and gene mutation status as independent variables. LGG was taken 823 as the reference tumour type. Only driver genes with $>250$ mutant samples in the dataset were included in the model.

Associations between copy number signature attribution (binarized to present or absent) and age at diagnosis (binarized to above or below median separately for each cancer type) were performed within cancer types using a two-sided Fisher's exact test ( $n=8,841$ across all cancer types). All p-values were corrected for multiple hypothesis testing using the Benjamini-Hochberg method and only associations with

831 both $q<0.05$ and $|\log 2(O R)|>1$ were reported. 
834 Copy number signatures and defective homologous recombination

835 Signatures were tested for enrichment in tumour types using one-sided Mann-

836 Whitney tests of signature attribution in a given tumour type versus all other tumour

837 types. This was performed for all signature and tumour combinations. All p-values

838 were corrected for multiple hypothesis testing using the Benjamini-Hochberg

839 method.

840

841 Core homologous recombination (HR) repair pathway member genes were chosen

842 to interrogate: $B R C A 1, B R C A 2, R A D 51 C, P A L B 2^{64,65}$. Copy number alterations across these genes were identified based on ASCAT copy number profiles for

844 homozygous deletions (i.e., $\mathrm{CN}=\{0,0\}$ ) and $\mathrm{LOH}$ (i.e., $\mathrm{CN}=\{>0,0\}$ ). Somatic SNVs

845 and indels were taken from Ref. ${ }^{63}$. Pathogenic germline variants in BRCA1 and

846 BRCA2 were taken from Ref. ${ }^{66}$. Samples were deemed as bi-allelically mutated for

847 the HR pathway if homozygously deleted (HD) or if $>1$ of any of the other classes of

848 alteration were present within any of the HR pathway genes. Mono-allelic loss was

849 defined as 1 of any of the non-HD alterations within any of the HR pathway genes.

850 Wildtype was defined as no alterations in any HR pathway genes. The associations

851 between HR pathway status and CN15 were then restricted to only breast $(n=589)$,

852 ovarian ( $n=309)$, and pan-cancer $(n=4,919)$. Two-sided fisher's exact tests were

853 performed between wild-type and mono-allelic samples, between wild-type and bi-

854 allelic samples, and between mono-allelic and bi-allelic HR pathway status samples.

855 All p-values were corrected for multiple hypothesis testing using the Benjamini-

856 Hochberg method. 
858 Copy number signatures associated with changes of overall survival

859 Survival data for 11,160 TCGA patients were obtained from the TCGA Clinical data

860 Resource R package ${ }^{67}$. Univariate disease specific survival analysis for signatures

861 was performed using a log-rank test and Kaplan-Meier curves in R, with groups

862 being unattributed (attribution $=0$ ) and attributed (attribution $>0$ ) for each signature

863 separately, or for summed attributions of a set of signatures (e.g., amplicon

864 signatures).

865

866 Multivariate disease-specific survival analysis was performed using the Cox's

867 proportional hazards model in $\mathrm{R}$ with Boolean attributed/non-attributed variables for each copy number signature and tumour type as covariates. To account for potential violations of Cox's model's proportional hazards assumption, we also conducted the same analysis using the accelerated failure time model with the Weibull distribution using the flexsurvreg function in R. All p-values were corrected for multiple hypothesis testing using the Benjamini-Hochberg method.

873

$874 \quad$ Simulating copy number profiles

875 Simulation framework: Genomes were initialized as 23 pairs of individual

876 chromosomes, with lengths corresponding to those seen in the human genome,

877 where the $23^{\text {rd }}$ pair could be either $X, X$ or $X, Y$. Each chromosome was initialized as 878 a data table with chromosome (1-22, X, Y), start position, end position, and allele 879 (either A or B). Genomic events were recorded as altering one of these data tables in 880 the appropriate way, adding or removing segments as necessary. Gains and losses:

881 The $\log _{10}($ size) of sub-chromosomal gains were drawn from a Gaussian mixture with 882 components: 


$$
\mathbf{N}\left(\mu=5.961351, \sigma^{2}=0.4199448\right)
$$

$$
\mathbf{N}\left(\mu=7.786183, \sigma^{2}=0.1068539\right)
$$

885 at proportions $p_{1}=0.7360366$ and $p_{2}=1-p_{1}$. The $\log _{10}($ size $)$ of sub-chromosomal

886 losses were drawn from a gaussian mixture with components:

$$
\mathbf{N}\left(\mu=6.188331, \sigma^{2}=0.5686788\right)
$$

$$
\mathbf{N}\left(\mu=7.588125, \sigma^{2}=0.1326166\right)
$$
$(\mathrm{CN} 1+\mathrm{CN} 9$ attribution $>0.8)$ from segments that were copy number 1 for the loss estimated using a Gaussian mixture model on the $\log _{10}$ (sizes) of the appropriate segments with two components due to the bimodal nature of the segment length distributions.

896

897 First the chromosome on which the gain/loss will occur is randomly sampled with 898 probabilities $1 / n$, where $n$ is the number of separate chromosomes in the current genome. The event size, $\lambda$, is then drawn from the previously stated multinormal

900 distributions; if an event size greater than the chromosomal size is drawn, then a

901 new size is drawn. The start of the event, $b_{1}$, is then drawn from a uniform

902 distribution,

$$
b_{1} \sim \mathbf{U}(1, e-\lambda)
$$

904 where $e$ is the cumulative length of the chosen chromosome, and the end of the event, $b_{2}=b_{1}+\lambda$. 
907 Gains are treated as tandem duplications, so that the gained region is inserted

908 immediately after the start breakpoint. On unaltered chromosome, this will alter the

909 chromosome from a single segment with start=1 and end=e to a chromosome with

910 four segments, with starts $=\left[1, b_{1}+1, b_{1}+1, b_{2}+1\right]$ and ends $=\left[b_{1}, b_{2}, b_{2}, e\right]$, each with the

911 chosen chromosome identity and allele; note that this will eventually lead to a copy

912 number profile with 3 segments with starts $==\left[1, b_{1}+1, b_{2}+1\right]$ and ends $=\left[b_{1}, b_{2}, e\right]$. A loss

913 will instead lead to a chromosome with two segments with starts $=\left[1, b_{2}\right]$ and

914 ends $=\left[b_{1}, e\right]$.

915

916 Simulating chromothripsis: For chromothriptic events, the $\log _{10}$ (number of segments)

917 for the resulting chromosome is drawn from a normal distribution:

$$
n \sim \mathbf{N}(\mu=1.3, \sigma=0.3),
$$

919 while the $\log _{10}$ (length) of segments are drawn from a normal distribution

$$
\lambda \sim \mathbf{N}(\mu=6, \sigma=0.7),
$$

and the start of the chromothriptic event is drawn from a uniform distribution:

$$
\mathbf{U}\left(1, e-\sum_{1}^{n} \lambda_{n}\right)
$$

where $e$ is the size of the chromosome. The parameters for the distributions were chosen to match the empirical distributions observed in TCGA chromosomes that were called as chromothriptic in the PCAWG dataset.

927 The breakpoints of the chromothriptic event, $\left[b_{1}, \ldots, b_{n-1}\right]$, are then the cumulative sums of the segment sizes, apart from the first breakpoint which is 1 . The chromosome is then broken into $n$ segments by their cumulative lengths, defined by the breakpoints. Whether to lose a segment is drawn from a binomial distribution:

$$
\delta_{x} \sim \operatorname{Binom}(1,0.5)
$$


932 All segments were removed where $\delta_{x}=1$. The remaining segments were then

933 randomly reversed if:

$$
\rho_{x} \sim \operatorname{Binom}(1,0.5)=1
$$

935 Lastly, the remaining segments were resampled without replacement so that their

936 order is randomized, and are then concatenated together. The chromothriptic

937 chromosome replaces the original chromosome that it originates from.

938

939 Genome doubling and chromosomal gains/losses: All chromosomes in the set of

940 chromosomes are duplicated to simulate genome doubling. For chromosomal gains,

941 a single chromosome is duplicated, whereas for chromosomal losses a single

942 chromosome is removed.

944 Calculating copy number: Once an assortment of chromosomes has been simulated

945 from a mixture of the previously described processes, the combined copy number

946 across all derivative chromosomes must be calculated across the reference genome.

947 For each reference chromosome, $x$, all segments across the derivative

948 chromosomes that derive from $x$ are collated, and the breakpoints across $x$ are

949 defined as the ordered unique set of start or end positions of those segments. Then

950 the copy number for segment $i_{x}$, is calculated for each allele separately; the A allele

951 copy number is the count of A allele segments in all derivative chromosomes that

952 overlap the segment defined between $b_{i, x}$ and $b_{i+1, x}$, and similar for the B allele copy

953 number. Combined across all reference chromosomes, this gives an allele-specific

954 copy number profile. 
956 Combinations of simulations: The following simulations were performed, for 100

957 samples each:

$958 \quad$ CINx10 - 10 random gain or loss events.

$959 \quad$ CINx50 - 50 random gain or loss events.

960 - CINx10->WGD - 10 random gain or loss events, followed by WGD.

961 - CINx50->WGD - 50 random gain or loss events, followed by WGD.

962 - CINx5->WGD->CINx50 - 5 random gain or loss events, followed by WGD,

963 followed by 50 random gain or loss events.

- CINx5->WGD->CINx25->WGD->CINx25 - 5 random gain or loss events, followed by WGD, followed by 25 random gain or loss events, followed by WGD, followed by 25 random gain or loss events.

- Chromo. - Chromothripsis of a random chromosome.

- Chromo.->WGD - Chromothripsis of a random chromosome, followed by WGD.

- Chromo.->Amp. - Chromothripsis of a random chromosome, followed by chromosomal gain of the derivative chromothriptic chromosome.

- Chromo.->Amp.->WGD - Chromothripsis of a random chromosome, followed by chromosomal gain of the derivative chromothriptic chromosome, followed

- Chromo.->Amp.x5->WGD. Chromothripsis of a random chromosome, followed by chromosomal gain of the derivative chromothriptic chromosome five times, followed by WGD.

978 For random gain/loss events, a binomial draw was used to decide whether a gain or 979 loss occurred, with $p_{\text {gain }}=0.4$. 
982

983

984

985

986

987

988

989

990

991

992

993

994

995

996

997

998

999

1000

1001

1002

1003

1004

1005

1006

1007

1008

1009

1010

1011

1012

1013

1014

1015

1016

1017

1018

1019

1020

1021

1022

1023

1024

1025

1026

1027

1028

1029

1030

1031

\section{REFERENCE}

1. Hanahan, D. \& Weinberg, R. A. Hallmarks of cancer: The next generation. Cell 144, 646-674 (2011).

2. Davoli, T., Uno, H., Wooten, E. C. \& Elledge, S. J. Tumor aneuploidy correlates with markers of immune evasion and with reduced response to immunotherapy. Science (80-. ). 355, (2017).

3. Taylor, A. M. et al. Genomic and Functional Approaches to Understanding Cancer Aneuploidy. Cancer Cell 33, 676-689 e3 (2018).

4. Ben-David, U. \& Amon, A. Context is everything: aneuploidy in cancer. Nature Reviews Genetics 21, 44-62 (2020).

5. Rajagopalan, H. \& Lengauer, C. Aneuploidy and cancer. Nature 432, 338-341 (2004).

6. Beroukhim, R. et al. The landscape of somatic copy-number alteration across human cancers. Nature 463, 899-905 (2010).

7. Sansregret, L. \& Swanton, C. The role of aneuploidy in cancer evolution. Cold Spring Harbor Perspectives in Medicine 7, (2017).

8. Bielski, C. M. et al. Genome doubling shapes the evolution and prognosis of advanced cancers. Nat. Genet. 50, 1189-1195 (2018).

9. Stephens, P. J. et al. Massive genomic rearrangement acquired in a single catastrophic event during cancer development. Cell 144, 27-40 (2011).

10. Bolhaqueiro, A. C. F. et al. Ongoing chromosomal instability and karyotype evolution in human colorectal cancer organoids. Nat. Genet. 51, 824-834 (2019).

11. Shoshani, O. et al. Chromothripsis drives the evolution of gene amplification in cancer. Nature 1-5 (2020). doi:10.1038/s41586-020-03064-z

12. Umbreit, N. T. et al. Mechanisms generating cancer genome complexity from a single cell division error. Science (80-. ). 368, (2020).

13. Alexandrov, L. B. et al. Signatures of mutational processes in human cancer (vol 500, pg 415, 2013). Nature 502, (2013).

14. Alexandrov, L. B. et al. The repertoire of mutational signatures in human cancer. Nature 578, 94-101 (2020).

15. Alexandrov, L. B. et al. Clock-like mutational processes in human somatic cells. Nat Genet 47, 1402-1407 (2015).

16. Gulhan, D. C., Lee, J. J., Melloni, G. E. M., Cortes-Ciriano, I. \& Park, P. J. Detecting the mutational signature of homologous recombination deficiency in clinical samples. Nat Genet 51, 912-919 (2019).

17. Behjati, S. et al. Mutational signatures of ionizing radiation in second malignancies. Nat. Commun. 7, (2016).

18. Alexandrov, L. B. et al. Mutational signatures associated with tobacco smoking in human cancer. Science 354, 618-622 (2016).

19. Haradhvala, N. J. et al. Distinct mutational signatures characterize concurrent loss of polymerase proofreading and mismatch repair. Nat Commun 9, 1746 (2018).

20. Kucab, J. E. et al. A Compendium of Mutational Signatures of Environmental Agents. Cell 177, 821-836 e16 (2019).

21. Meier, B. et al. Mutational signatures of DNA mismatch repair deficiency in C. elegans and human cancers. Genome Res 28, 666-675 (2018).

22. Pich, O. et al. The mutational footprints of cancer therapies. Nat Genet 51, 1732-1740 (2019).

23. Steele, C. D. et al. Undifferentiated Sarcomas Develop through Distinct 
Evolutionary Pathways. Cancer Cell 35, 441-456.e8 (2019).

24. Macintyre, G. et al. Copy number signatures and mutational processes in ovarian carcinoma. Nat Genet 50, 1262-1270 (2018).

25. Pladsen, A. V. et al. DNA copy number motifs are strong and independent predictors of survival in breast cancer. Commun. Biol. 3, 1-9 (2020).

26. Ashiqul Islam, S. M. et al. Uncovering novel mutational signatures by de novo extraction with. bioRxiv 2020.12.13.422570 (2020). doi:10.1101/2020.12.13.422570

27. Alexandrov, L. B. \& Stratton, M. R. Mutational signatures: The patterns of somatic mutations hidden in cancer genomes. Current Opinion in Genetics and Development 24, 52-60 (2014).

28. Koh, G., Zou, X. \& Nik-Zainal, S. Mutational signatures: Experimental design and analytical framework. Genome Biology 21, 37 (2020).

29. López, S. et al. Interplay between whole-genome doubling and the accumulation of deleterious alterations in cancer evolution. Nat. Genet. 52, 283-293 (2020).

30. Davoli, T. et al. Cumulative haploinsufficiency and triplosensitivity drive aneuploidy patterns and shape the cancer genome. Cell 155, 948-962 (2013).

31. Mertens, F., Johansson, B., Höglund, M. \& Mitelman, F. Chromosomal Imbalance Maps of Malignant Solid Tumors: A Cytogenetic Survey of 3185 Neoplasms. Cancer Res. 57, 2765-2780 (1997).

32. Kim, H. et al. Extrachromosomal DNA is associated with oncogene amplification and poor outcome across multiple cancers. Nat. Genet. 52, 891897 (2020).

33. Zheng, S. et al. Comprehensive Pan-Genomic Characterization of Adrenocortical Carcinoma. Cancer Cell 30, 363 (2016).

34. Davis, C. F. et al. The somatic genomic landscape of chromophobe renal cell carcinoma. Cancer Cell 26, 319-330 (2014).

35. Menghi, F. et al. The Tandem Duplicator Phenotype Is a Prevalent GenomeWide Cancer Configuration Driven by Distinct Gene Mutations. Cancer Cell 34, 197-210.e5 (2018).

36. Bielski, C. M. et al. Genome doubling shapes the evolution and prognosis of advanced cancers. Nat Genet 50, 1189-1195 (2018).

37. Lo, A. W. I. et al. DNA amplification by breakage/fusion/bridge cycles initiated by spontaneous telomere loss in a human cancer cell line. Neoplasia 4, 531538 (2002).

38. Deshpande, V. et al. Exploring the landscape of focal amplifications in cancer using AmpliconArchitect. Nat. Commun. 10, 1-14 (2019).

39. Consortium, I. T. P.-C. A. of W. G. Pan-cancer analysis of whole genomes. Nature 578, 82-93 (2020).

40. Behjati, S. et al. Recurrent mutation of IGF signalling genes and distinct patterns of genomic rearrangement in osteosarcoma. Nat. Commun. 8, (2017).

41. Cortes-Ciriano, I. et al. Comprehensive analysis of chromothripsis in 2,658 human cancers using whole-genome sequencing. Nat Genet (2020). doi:10.1038/s41588-019-0576-7

42. Furgason, J. M. et al. Whole genome sequence analysis links chromothripsis to EGFR, MDM2, MDM4, and CDK4 amplification in glioblastoma. Oncoscience 2, 618-628 (2015).

43. Hoadley, K. A. et al. Cell-of-Origin Patterns Dominate the Molecular Classification of 10,000 Tumors from 33 Types of Cancer. Cell 173, 291-304 
1082

1083

1084

1085

1086

1087

1088

1089

1090

1091

1092

1093

1094

1095

1096

1097

1098

1099

1100

1101

1102

1103

1104

1105

1106

1107

1108

1109

1110

1111

1112

1113

1114

1115

1116

1117

1118

1119

1120

1121

1122

1123

1124

1125

1126

1127

1128

1129

1130

1131

e6 (2018).

44. Knudson, A. G. Hereditary cancer: two hits revisited. J Cancer Res Clin Oncol 122, 135-140 (1996).

45. Scarpa, A. et al. Whole-genome landscape of pancreatic neuroendocrine tumours. Nature 543, 65-71 (2017).

46. Ricketts, C. J. et al. The Cancer Genome Atlas Comprehensive Molecular Characterization of Renal Cell Carcinoma. Cell Rep. 23, 313-326.e5 (2018).

47. Menghi, F. et al. The tandem duplicator phenotype as a distinct genomic configuration in cancer. Proc. Natl. Acad. Sci. U. S. A. 113, E2373-E2382 (2016).

48. McBride, D. J. et al. Tandem duplication of chromosomal segments is common in ovarian and breast cancer genomes. J. Pathol. 227, 446-455 (2012).

49. TCGA. Comprehensive and Integrated Genomic Characterization of Adult Soft Tissue Sarcomas. Cell 171, 950-965 e28 (2017).

50. Sakofsky, C. J. et al. Repair of multiple simultaneous double-strand breaks causes bursts of genome-wide clustered hypermutation. PLoS Biol. 17, e3000464 (2019).

51. Pfister, K. et al. Identification of Drivers of Aneuploidy in Breast Tumors. Cell Rep. (2018). doi:10.1016/j.celrep.2018.04.102

52. Schjølberg, A. R., Clausen, O. P. F., Burum-Auensen, E. \& De Angelis, P. M. Aneuploidy is associated with TP53 expression but not with BRCA1 or TERT expression in sporadic colorectal cancer. Anticancer Res. (2009).

53. Cazzola, A. et al. TP53 deficiency permits chromosome abnormalities and karyotype heterogeneity in acute myeloid leukemia. Leukemia (2019). doi:10.1038/s41375-019-0550-5

54. Thorsson, V. et al. The Immune Landscape of Cancer. Immunity 48, 812830.e14 (2018).

55. Tate, J. G. et al. COSMIC: the Catalogue Of Somatic Mutations In Cancer. Nucleic Acids Res. 47, D941-D947 (2019).

56. De Jonge, M. M. et al. Frequent homologous recombination deficiency in highgrade endometrial carcinomas. Clin. Cancer Res. 25, 1087-1097 (2019).

57. Davies, $\mathrm{H}$. et al. HRDetect is a predictor of BRCA1 and BRCA2 deficiency based on mutational signatures. Nat. Med. 23, 517-+ (2017).

58. Van Loo, P. et al. Allele-specific copy number analysis of tumors. Proc Natl Acad Sci U S A 107, 16910-16915 (2010).

59. Alexandrov, L. B. et al. Signatures of mutational processes in human cancer. Nature 500, 415-421 (2013).

60. Health, N. et al. Signatures of mutational processes in human cancer. Nature 1-108 (2013). doi:10.1038/nature

61. Liu, J. et al. An Integrated TCGA Pan-Cancer Clinical Data Resource to Drive High-Quality Survival Outcome Analytics. Cell 173, 400-416.e11 (2018).

62. Lowy, D. R., Kibbe, W. A., Ph, D., Staudt, L. M. \& Ph, D. New engla nd journal. 1109-1112 (2016).

63. Martincorena, I. et al. Universal Patterns of Selection in Cancer and Somatic Tissues. Cell 171, 1029-1041 e21 (2017).

64. Knijnenburg, T. A. et al. Genomic and Molecular Landscape of DNA Damage Repair Deficiency across The Cancer Genome Atlas. Cell Rep 23, 239-254 e6 (2018).

65. Nguyen, L., W. M. Martens, J., Van Hoeck, A. \& Cuppen, E. Pan-cancer landscape of homologous recombination deficiency. Nat. Commun. 11, 1-12 
(2020).

1134

66. Yost, S., Ruark, E., Alexandrov, L. B. \& Rahman, N. Insights into BRCA Cancer Predisposition from Integrated Germline and Somatic Analyses in 7632

1135 Cancers. JNCI Cancer Spectr. 3, (2019).

1136

1137

67. Liu, J. et al. An Integrated TCGA Pan-Cancer Clinical Data Resource to Drive High-Quality Survival Outcome Analytics. Cell 173, 400-416.e11 (2018).

1138

1139 
bioRxiv preprint doi: https://doi.org/10.1101/2021.04.30.441940; this version posted April 30, 2021. The copyright holder for this preprint (which was not certified by peer review) is the author/funder, who has granted bioRxiv a license to display the preprint in perpetuity. It is made available under aCC-BY-NC-ND 4.0 International license.

1141

1142 